\title{
Computational and Spectroscopic Tools for the Detection of Bond Covalency in Pu(IV) Materials
}

\author{
Paul S. Bagus,* Bianca Schacherl, and Tonya Vitova*
}

Cite This: Inorg. Chem. 2021, 60, 16090-16102

Read Online

ABSTRACT: Plutonium is used as a major component of new-generation nuclear fuels and of radioisotope batteries for Mars rovers, but it is also an environmental pollutant. Plutonium clearly has high technological and environmental importance, but it has an extremely complex, not wellunderstood electronic structure. The level of covalency of the $\mathrm{Pu} 5 \mathrm{f}$ valence orbitals and their role in chemical bonding are still an enigma and thus at the frontier of research in actinide science. We performed fully relativistic quantum chemical computations of the electronic structure of the $\mathrm{Pu}^{4+}$ ion and the $\mathrm{PuO}_{2}$ compound. Using four different theoretical tools, it is shown that the $5 \mathrm{f}$ orbitals have very little covalent character although the $5 \mathrm{f}(7 / 2) \mathrm{a}_{2 \mathrm{u}}$ orbital with the highest orbital energy has the greatest extent of covalency in $\mathrm{PuO}_{2}$. It is illustrated that the $\mathrm{Pu} \mathrm{M}_{4,5}$ edge high-energy resolution X-ray absorption near-edge structure ( $\mathrm{Pu} \mathrm{M} \mathrm{M}_{4,5}$ HR-XANES) spectra cannot be interpreted in terms of dipole selection rules applied between individual $3 \mathrm{~d}$ and $5 \mathrm{f}$ orbitals, but the selection rules must be applied between the total wavefunctions for the initial and excited states. This is because the states cannot be represented by single determinants. They are shown to involve major redistributions on the $5 f$ electrons over the different $5 \mathrm{f}$ orbitals. These redistributions could be viewed as shake-up-like excitations in the $5 f$ shell from the lowest orbital energy from $J=5 f(5 / 2)$ into higher orbital energy $J=5 f(7 / 2)$. We show that the second peak in the $\mathrm{Pu} \mathrm{M}_{4}$ edge and the high-energy shoulder of the $\mathrm{Pu} \mathrm{M}_{5}$ edge HR-XANES spectra probe the $5 f(7 / 2) \mathrm{a}_{2 \mathrm{u}}$ orbital; thus, these spectral features are expected to change upon bond variations. We describe theoretical and spectroscopy tools, which can be applied for all actinide elements in materials with cubic structure.

\section{INTRODUCTION}

The actinide elements are more than ever of high relevance for our everyday life in many different ways. One main application remains electricity production. Furthermore, we still need to solve challenges like building safe long-term repositories for high-level radioactive waste and developing effective remediation strategies for cleaning up polluted sites. Along with Ac225, some short-lived $\alpha$ emitters like Th-227 and U-230 show high promise for cancer treatment. ${ }^{1-3}$ The heat production of actinide $\alpha$-emitters like $\mathrm{Pu}-238$ is the driving force of our explorer on Mars - the Curiosity rover. Clearly, the actinide elements are of significance for our societal challenges. However, we still do not have very good understanding of their fascinating and complex electronic structure. Gaining a deeper understanding of how the actinides form chemical bonds and what is the role of their $5 \mathrm{f}, 6 \mathrm{~d}$, and $7 \mathrm{~s}$ valence electrons is the key, for example, for developing ligands with targeted properties for medical applications or for effective remediation strategies. It is still a great challenge and not completely understood why specific N-donor ligands have very high binding selectivity for actinides compared to the chemically similar lanthanides. This mechanistic understanding is essential for designing ligands for separation processes with the aim to reuse the actinides for new nuclear fuels or to reduce their radiotoxicity. There is limited experimental and theoretical evidence that the An(III) might form more covalent bonds compared to the $\operatorname{Ln}(\mathrm{III})$, and this can be the underlying mechanism of the selectivity. The combination of novel spectroscopy techniques and state-of-the-art quantum chemical computations give high promise for a thorough probe of the actinide electronic structure and bond covalency. In particular, we consider an analysis of the bond covalency combining novel theoretical and experimental methods to analyze the electronic structure of $\mathrm{PuO}_{2}$. These same methods can be applied to determine the covalent character of actinides in other materials.

In the present work, we apply four different $a b$ initio computational approaches to probe the covalency of the $\mathrm{Pu}-\mathrm{O}$ chemical bond. We also make a detailed comparison of $\mathrm{Pu} \mathrm{M}$ edge high-resolution $\mathrm{X}$-ray absorption near-edge structure (HR-XANES) spectra ${ }^{4-8}$ of $\mathrm{PuO}_{2}$ with rigorous theoretical calculations of the energies and intensities of the HR-XANES spectra (cf. refs 9-12). The main aim is to understand how we can extract chemical information and specifically insights into

Received: May 2, 2021

Published: October 11, 2021 
the $\mathrm{Pu}$-ligand bond covalency from these $\mathrm{Pu} \mathrm{M}_{4,5}$ absorption edges.

It is common to view XANES excitations in one-electron terms as a core electron being promoted into a specific valence level shell that is empty or not fully occupied. ${ }^{12}$ However, this is a simplification and the XANES transitions are between many-electron wavefunctions (WFs). It is necessary to take many-body effects into account to properly describe these WFs, for both the initial and excited states of the XANES transitions. A many-body effect that is quite important is the coupling of the spin and orbital angular momenta of the openshell electrons. On the basis of atomic models and formal algebraic analysis of the coupling, this has been considered for a range of systems by van der Laan and collaborators. ${ }^{13-15}$ However, these approaches neglect the formation of covalent bonds between the metal cation and the ligand anions and the ligand field splittings related to this covalency. It is possible to use crystal field theory to include the effects of covalency without an explicit treatment of the covalent mixing of cation and ligand character in the orbitals as was done, for example, by Butorin. ${ }^{16}$ However, it is useful to use a quantum mechanical theory that directly includes the covalent theory of the orbitals on an $a b$ initio basis and without empirical parameters. $A b$ initio approaches to the analysis of XANES, which explicitly include covalent mixing of the molecular orbitals are available and could be used. Such $a b$ initio treatments have been made by Neese and collaborators for compounds of lighter elements (see the representative ref 17) and by Autschbach and collaborators for actinide compounds (see the representative ref 11 ).

Our present work is different from these prior works ${ }^{11,17}$ in that our studies are based on the use of four-component relativistic orbitals based on solutions of the Dirac-Coulomb Hamiltonian ${ }^{18}$ for the XANES studies. Thus, the WFs naturally include a balanced treatment of ligand field covalency and spin-orbit splitting as well as the rigorous treatment of the open-shell angular momentum coupling. Our approach has previously been applied to the study of the XANES of $\mathrm{Fe}$ compounds $\left(\mathrm{FeCl}_{4}\right.$ and $\left.\mathrm{Fe}_{2} \mathrm{O}_{3}\right){ }^{19,20}$ In the present work, the importance of this angular momentum coupling is applied to the $\mathrm{Pu} \mathrm{M} \mathrm{M}_{4,5}$ XANES of $\mathrm{PuO}_{2}$. As in our previous XANES studies, ${ }^{19,20}$ our theoretical analysis involves comparing the properties of isolated atoms with those of the compound. Thus, we describe relativistic WFs for atomic $\mathrm{Pu}^{4+}$ and for cluster models of $\mathrm{PuO}_{2}$. By comparing the XANES for atomic models with that for $\mathrm{PuO}_{2}$, we are able to distinguish the XANES for the pure spin-orbit splitting in the atom to the spin-orbit splitting modified by ligand field splittings and covalent mixing between the $\mathrm{Pu}(5 f)$ and $\mathrm{O}(2 \mathrm{p})$ orbitals in the oxide. This allows us to predict the extent of the influence that the structural environment has on the $\mathrm{M}_{4,5}$ XANES of different $\mathrm{Pu}$ compounds which have the same oxidation state.

Since we focus on the angular momentum coupling, we do not include other many-body effects that are required to accurately describe the energies of different electronic multiplets; these are commonly described as dynamic correlation effects. $^{21,22}$ These correlation effects can be applied to the properties of heavy-metal compounds (cf. refs 11, 23-26) and have been used for the XANES edges of $\mathrm{PuO}_{2}{ }^{2+}$ (plutonyl). ${ }^{11}$ We have chosen not to use such methods in our study of $\mathrm{PuO}_{2}$. First because the treatment of dynamic correlation for a large number of multiplets, as required to study the XANES, is more complex when a fully relativistic treatment for the electronic structure is made. But more importantly, to examine the extent to which the inclusion of simply the angular momentum coupling of the open $f$ shell electron systems, a property that is necessary to properly describe the initial and excited multiplets, provides a useful description of the XANES of $\mathrm{PuO}_{2}$. We consider $\mathrm{PuO}_{2}$ as a representative case where our conclusions can, very likely, be extended to other heavy-metal oxides.

\section{CONCEPTS AND METHODS}

Angular Momentum Coupling. It is appropriate to start with the definition that we use for the WFs which are the basis of our theoretical analysis of the $\mathrm{Pu} \mathrm{M}_{4,5}$ XANES spectra. The multiplet is a collection of degenerate states, which satisfy the orbital and spin angular momentum coupling of the open-shell electrons (cf. refs 27, 28 ). For an isolated atom, the appropriate quantum numbers ${ }^{27,28}$ are $\mathrm{S}$ for the total spin, $\mathrm{L}$ for the total orbital angular momentum, and $\mathrm{J}$ for the sum where the vector relationship $\mathrm{J}=\mathrm{L}+\mathrm{S}$ holds. For Russell-Saunders (RS) coupling, the multiplets are denoted ${ }^{2 S+1} \mathrm{~L}_{\mathrm{J}}$. Details of the RS multiplets including details of the coupling, the degeneracies, and the energetic ordering can be found in standard texts, for example, refs 28, 29. For the RS multiplets of other symmetries besides the spherical symmetry of isolated atoms, the spin remains coupled to $S$ but the orbital angular momentum, modified by ligand field splittings, is rigorously one of the representations of the appropriate point group. ${ }^{30}$ However, we still describe this as an angular momentum coupling since the orbital angular momentum remains a significant part of the open-shell coupling. To couple the $S$ with the orbital symmetry to determine a total angular momentum for these lower-symmetry RS multiplets, there are rules based on the symmetries of double groups. ${ }^{30}$

When spin-orbit coupling is taken fully into account, the total spin angular momentum and the total orbital angular momentum are no longer good quantum numbers since different RS multiplets with the same total angular momenta may mix. ${ }^{30}$ For the isolated cation, the $J$ value is still a good quantum number. For the octahedral double group of $\mathrm{PuO}_{2}, \mathrm{O}_{\mathrm{h}}{ }^{*},{ }^{31}$ and with the Bethe notation, ${ }^{32}$ the symmetries that remain good properties are: For systems with even numbers of electrons, these are the singly degenerate $\Gamma_{1}$ and $\Gamma_{2}$, the doubly degenerate $\Gamma_{3}$, and the triply degenerate $\Gamma_{4}$ and $\Gamma_{5}$. For systems with odd numbers of electrons, they are the doubly degenerate $\Gamma_{6}$ and $\Gamma_{7}$, and the fourfold degenerate $\Gamma_{8}$. The double-group symmetries for the electron orbitals are typically denoted with lower case, $\gamma_{6}, \gamma_{7}$, and $\gamma_{8}$. We will make limited use of these double-group notations since they are not especially familiar and there is limited agreement on their notation. ${ }^{32,33}$ However, it is convenient to use $\gamma_{6}, \gamma_{7}$, and $\gamma_{8}$ to distinguish the spin-orbit and ligand field split orbitals of $\mathrm{PuO}_{2}$.

In Figure $1 \mathrm{~A}$, we show a schematic of how the $\mathrm{Pu} 5 \mathrm{f}$ orbitals are split between the pure atomic spin-orbit splittings of $5 \mathrm{f}_{5 / 2}$ and $5 \mathrm{f}_{7 / 2}$ (left) and the pure ligand field splittings of $t_{2 w}, t_{1 w}$ and $a_{2 u}$ (right); in the middle, the double-group symmetry orbitals, $\gamma_{6 u}, \gamma_{7 w}$ and $\gamma_{8 w}$ are shown where the dotted lines indicate the dominant origins of the double-group orbitals. The inset in Figure 1A shows the orbital energies of the open-shell orbitals from our relativistic calculation of the $\mathrm{PuO}_{2} \mathrm{WF}$. The figure clearly shows the interplay of spin-orbit and ligand field splittings, which significantly modifies the orbital splitting.

These spin-orbit and ligand field split $5 f$ orbitals are occupied in different ways in determinants which are the basis of the $\mathrm{N}$-electron multiplet WFs. Depending on whether spin-orbit and ligand field splittings are included, the degeneracies and the energy separations of the multiplets are different. The different couplings for the isolated $\mathrm{Pu}^{4+}$ cation and $\mathrm{PuO}_{2}$ are discussed in the Wavefunctions for the Initial and Excited States section. There, we distinguish between the cases of RS, $j-j$, and intermediate coupling. RS, appropriate for low effective nuclear charge, is where the spins and the orbital angular momenta are coupled together separately. While $j-j$ coupling, appropriate for high effective nuclear charge, is where the multiplets are dominated by a single occupation of the spin-orbit split open- 

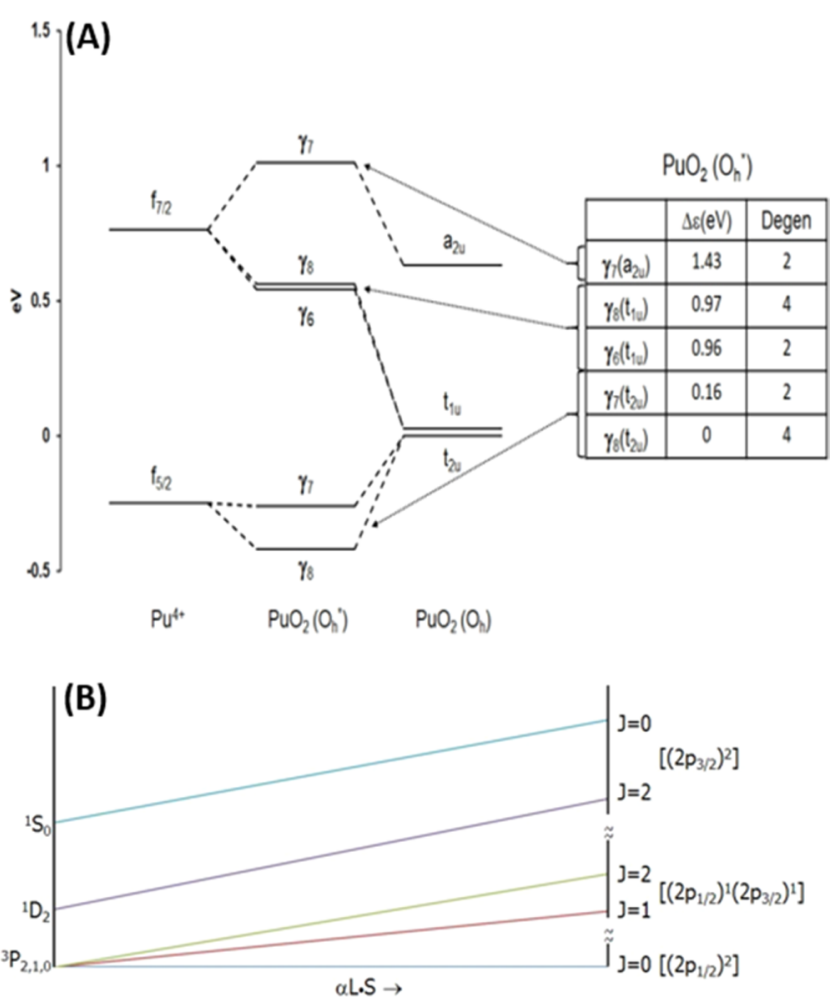

Figure 1. (A) Schematic representation of the spin-orbit and ligand field splittings of the dominantly $\mathrm{Pu} 5 \mathrm{f}$ open-shell orbitals for the $\mathrm{PuO}_{2}$ cluster WF. The dominant relationships are indicated by the dashed lines connecting the extremes of pure spin-orbit and pure ligand field splittings. (B) Example of RS and $j-j$ coupling for a model of six-electron atoms with configuration $1 \mathrm{~s}^{2} 2 \mathrm{~s}^{2} 2 \mathrm{p}^{2}$; the $x$-axis is the magnitude of the $\mathrm{L}-\mathrm{S}$ coupling; the $y$-axis is the energy separation of the multiplets.

shell spinors. This is shown schematically in Figure 1B for a model of six-electron atoms with configuration $1 s^{2} 2 s^{2} 2 p^{2}$ from low $Z$ ions to high $Z$ ions. The $x$-axis is the magnitude of the $\mathrm{L}-\mathrm{S}$ coupling and is labeled $\propto \mathrm{L} \cdot \mathrm{S}$, which is closely related to the effective charge. The $y$ axis is the energy separation of the multiplets. At low $Z$, Hund's rules $^{27}$ hold and the lowest multiplets are ${ }^{3} \mathrm{P}$ with, at $Z-6$, the different $\mathrm{J}$ levels separated by $\sim 20 \mathrm{~cm}^{-1}$ For high $Z$ in Figure $1 \mathrm{~B}$ (right), the multiplets are essentially pure $j-j$ coupled with the $2 p_{1 / 2}$ and $2 p_{3 / 2}$ occupations shown in the figure. The separation of the different levels becomes huge when $j-j$ coupling is appropriate. Around $Z=92$, the separation of the lowest and highest levels is several thousand electron volts. Furthermore, the high $Z$ multiplets are now linear combinations of the RS multiplets. For example, the lowest $J=0$ levels in the high $\mathrm{Z}$ limit, $\left(2 \mathrm{p}_{1 / 2}\right)^{2}$, are $2 / 3\left({ }^{3} \mathrm{P}_{0}\right)$ and $1 / 3\left({ }^{1} \mathrm{~S}_{0}\right)$. Below, we apply the concepts described above and in ref 34 to describe the intermediate coupling for the initial and excited multiplets of interest here. In particular, for the intermediate coupled multiplets, we determine the fractional composition in terms of the RS multiplets.

Clusters and Wavefunctions. The WFs and properties of $\mathrm{PuO}_{2}$ are represented with two models. To understand the consequences of pure spin-orbit splitting on the $\mathrm{M}_{4,5}$ edge XANES, we consider an isolated $\mathrm{Pu}^{4+}$ cation. In addition, to take into account the ligand field splittings, $\mathrm{PuO}_{2}$ is represented by an embedded cluster model with a central $\mathrm{Pu}$ cation and its eight nearest $\mathrm{O}$ anion neighbors, $\mathrm{PuO}_{8}$, in the experimental fluorite geometry. ${ }^{31}$ This cluster is embedded in a large number of point charges with the nominal $\mathrm{Pu}$ and $\mathrm{O}$ ionicities to represent the effects of the extended Madelung potential. For details of the choices of cluster model and embedding, see, for example, refs 35,36 . The atoms and point charges are placed at crystal lattice sites for fluorite $\mathrm{PuO}_{2} \cdot{ }^{31} \mathrm{WFs}$ for the isolated cation and for the cluster are then determined for both the initial state configuration and the $M_{4,5}$ excited-state configuration, where a Pu electron is promoted from the $3 \mathrm{~d}$ to the $5 \mathrm{f}$ shell, as solutions of suitable Dirac-Coulomb Hamiltonians. ${ }^{37}$ The orbitals are four-component solutions of Dirac-Hartree-Fock (DHF) equations. While it is possible to work directly in a two-component formulation (cf. refs 23-26), we have preferred to retain the small components to avoid any uncertainties with direct calculations in a two-component formalism. Since the approximations in the two-component formulations are not of direct relevance to our study of covalency in $\mathrm{PuO}_{2}$, we do not make an extensive comparison with such methods. However, one measure of the importance of directly including the small components, especially for $\mathrm{Pu}$, is the Mulliken population ${ }^{38,39}$ of these components. For the ground-state DHF orbitals of the embedded $\mathrm{PuO}_{8}$ cluster, the small-component $\mathrm{Pu}$ population is 0.79 electrons, which is not entirely negligible. Of course, most of this population is for the $\mathrm{Pu}$ core orbitals and not the valence orbitals, which explains the success of using two-component models. A more detailed comparison is beyond the scope of this paper.

It is important to stress that the orbitals are different for the initial state configuration, where the core shell is filled, and the excited configurations, where an electron has been promoted from the $\mathrm{M}_{4}$ or $\mathrm{M}_{5}$ core shell to the $5 \mathrm{f}$ shell. Specifically, they are variationally optimized for each of the different configurations. This is done to take account, in a compact way, of the screening in response to the core hole; see the discussion of this treatment of screening in refs 36,40 . The WFs are sums over determinants, described as configuration interaction (CI). The set of determinants summed over have all possible distributions of the electrons within the orbital of each open shell; these WFs correctly represent the spin and orbital angular momentum coupling of the open-shell electrons and have the correct degeneracies of the multiplets. ${ }^{36,40}$ The calculations of the orbitals and WFs were performed with the Dirac program system; ${ }^{18,41}$ a complete description of these calculations including the parameters for the basis functions, the choice of determinants, and the inclusion or neglect of spin-orbit coupling is given in the Supporting Information.

Methods of Analysis of Orbitals and Bond Covalency. To understand the significance of the theoretical results for the XANES spectra, we use several novel methods to analyze the properties and character of the orbitals and WFs. These methods are described briefly below; for further details, see refs 36, 40, 42-44 and the Supporting Information.

For the orbitals, an important concern is the degree of covalency, which arises from the mixing of the central metal and $\mathrm{O}$ orbitals. In particular, for the closed-shell orbitals, which are dominantly $\mathrm{O}$, there is a bonding mixing with the frontier orbitals of the metal, and for the open-shell, frontier metal orbitals, there is an antibonding with the $\mathrm{O}(2 \mathrm{p})$ orbitals. ${ }^{36,40,45-47}$ It is important to stress that while covalency is a very useful concept for understanding the chemical interactions in a compound, it has been difficult to rigorously define the extent of the covalency. The covalent character is the mixing of cation and ligand orbitals, but for the variational optimized orbitals of a compound, it is not possible to uniquely separate the contributions of the cation and the ligands. Methods related to Mulliken population analyses have been used to describe the covalency, but they give at best a qualitative view, cf. refs $43,44,48$, for a detailed discussion of these difficulties and for proposed alternatives. In the present work, as described below, we use four novel methods to obtain reliable and consistent measures of the extent of the covalent character for the initial and the $\mathrm{M}_{4,5}$ excited configurations of $\mathrm{PuO}_{2}$.

For $\mathrm{PuO}_{2}$, it is the covalency of the $5 \mathrm{f}$ orbitals that is of primary interest here. In particular, there is an important concern for whether the $5 f$ covalency changes between the initial, ground state, and the final excited $\mathrm{M}_{4,5}$ edge, XANES states. Such changes are quite common for the ionic states in X-ray photoemission spectroscopy (XPS), and this requires that care must be taken to distinguish what are described as initial and final state effects; see, for example, refs 36, 40. These effects must be separated to draw inferences from XPS about the ground-state electronic structure. Clearly, changes in the covalency between the ground state and the XANES excited states will 
provide useful information about whether a separation of initial and final state effects is also needed to interpret XANES data.

The four methods that we use to quantify the covalent character are as follows:

(1) The covalency contributes to differences in the orbital energies of the different spinors of the $\mathrm{PuO}_{2} 5 \mathrm{f}$ shell. This can be immediately seen in Figure 1A (right), where $\varepsilon\left(\mathrm{a}_{2 \mathrm{u}}\right)$ is $\sim 0.5 \mathrm{eV}$ higher, less bound, than $\varepsilon\left(\mathrm{t}_{1 \mathrm{u}}\right)$ and $\varepsilon\left(\mathrm{t}_{2 \mathrm{u}}\right)$. This difference in the orbital energies indicates that $\mathrm{a}_{2 \mathrm{u}}$ has a larger antibonding character than the $t_{1 u}$ and $t_{2 u}$ orbitals. Of course, there is also coupling with the spin-orbit splitting, and this can be seen in Figure 1A (middle) where the spin-orbit coupling is now added to the pure ligand field splitting of the right-hand-side panel.

(2) A second measure of the covalency of an orbital arises from the size of the orbital, which provides a direct measure of the extent of covalency. For the size, the orbital expectation value of $r$ measured with respect to the $\mathrm{Pu}$ nucleus, is used. Specifically, we define $r_{\text {eff }}$ as

$$
r_{\text {eff }}=\left[\left\langle r^{2}\right\rangle\right]^{1 / 2}
$$

The $\mathrm{O}$ contribution to a covalent $5 \mathrm{f}$ orbital is centered about the $\mathrm{O}$ atoms which are distant from Pu by over $2 \AA$, which is $\sim 3$ times larger than the $r_{\text {eff }}$ value of a $5 f$ orbital of a Pu cation (see below and ref 49). Thus, when one of the 5 f orbitals has a value of $r_{\text {eff }}$ larger than that of another $5 f$ orbital, then this orbital should have a larger covalent character.

(3) A third measure of the covalent character is obtained by projecting the orbitals of an isolated $\mathrm{Pu}$ cation on the $\mathrm{PuO}_{8}$ cluster orbitals. ${ }^{44,48}$ This can again be understood from Figure 1A. The orbitals isolated on the left of the schematic are pure atomic Pu $5 f$ orbitals, but the orbitals for the compound are not pure but contain some amount of $\mathrm{O}(2 \mathrm{p})$ character. The problem is to determine the extent of this mixture of $\mathrm{O}$ character into the open-shell $\mathrm{Pu} 5 \mathrm{f}$ orbital. It is reasonably common to estimate this character with a population analysis; see, for example, ref 28 , but such populations may be misleading. ${ }^{44}$ On the other hand, projections identify the atomic character through overlap integrals between the orbitals of a compound and those of the isolated atomic ion. ${ }^{44}$ Especially for relatively localized orbitals like the $\mathrm{Pu}(5 \mathrm{f})$, the projections provide useful guides to the covalent character and to the trends of the covalency between different orbitals and different compounds. ${ }^{44,48}$ The projections, $N_{\mathrm{P}}$, of interest here are $N_{\mathrm{P}}\left(5 f_{5 / 2}\right)$ and $N_{\mathrm{P}}\left(5 f_{7 / 2}\right)$, where sums are made over the six or eight components of the atomic shells. Sums may also be made over groups of cluster orbitals; e.g., all of the closed-shell orbitals. When the projections are made on a single compound orbital, the values of $N_{\mathrm{P}}$ for this orbital are $0 \leq N_{\mathrm{P}} \leq 1$, where $N_{\mathrm{P}}=0$ indicates that the orbital has no Pu character and $N_{\mathrm{P}}=$ 1 indicates that the orbital is a pure $\mathrm{Pu}$ orbital with no ligand character.

(4) The covalent mixing has been viewed as being driven by the overlap of the frontier orbitals of the isolated $\mathrm{Pu}^{4+}$ cation, the $5 f$ orbitals, and the isolated $\mathrm{O}^{2-}$ ligand anion, the $2 \mathrm{p}$ orbitals, modified by the energy splitting of these orbitals. ${ }^{50}$ We have examined these overlaps to identify the extent that they are consistent with the measures that we have described above. For the $\mathrm{O}$ orbitals, we have used $\mathrm{O}^{-}$orbitals since they are more diffuse than neutral $\mathrm{O}$ orbitals and they have a similar size to the orbitals of the oxides. ${ }^{51,52}$ For the $\mathrm{Pu} 5 \mathrm{f}$ and $\mathrm{O}$ fragment orbitals, we used linear combinations to have $\mathrm{O}_{h}$ symmetry orbitals that transformed as $t_{1 w}, t_{2 w}$ and $a_{2 u}$. For these $\mathrm{O}_{\mathrm{h}}$ symmetry orbitals, we computed the overlap $\mathrm{S}^{2}(\mathrm{i})$ where

$$
S^{2}(i)=\left\langle\mathrm{O}_{8}(2 \mathrm{p}, i) \mid \mathrm{Pu}(5 \mathrm{f}, i)\right\rangle^{2}
$$

The square of the overlap is used in eq 2 to avoid artificial changes of sign since the phases of the orbitals are not defined within a sign.
We have not considered the covalent character of the $\mathrm{O}(2 \mathrm{~s})$ or the $\mathrm{Pu}(6 \mathrm{~d})$. For the $\mathrm{O}(2 \mathrm{~s})$, we have examined the projection of the cation $\mathrm{Pu}(5 \mathrm{f})$ orbitals on the $\mathrm{PuO}_{8}$ cluster orbitals that are dominantly of $\mathrm{O}(2 \mathrm{~s})$ character as shown by their orbital energies. While these projections are not exactly zero, they are small. In particular, the sum of the magnitudes of the $5 f$ projections on the $\mathrm{O}(2 \mathrm{~s})$ orbitals is considerably smaller than the sum of the magnitudes of the $5 \mathrm{f}$ projections on the $\mathrm{O}(2 \mathrm{p})$ cluster orbitals. Thus, the dominant covalent character of the $5 f$ orbitals arises from mixing with the $\mathrm{O}(2 \mathrm{p})$ orbitals and the $\mathrm{O}(2 \mathrm{~s})$ can be safely neglected. There is considerable covalency of $\mathrm{Pu}(6 \mathrm{~d})$ in the gerade closed-shell orbitals of $\mathrm{PuO}_{2}$ The $\mathrm{U}(6 \mathrm{~d})$ covalency in $\mathrm{U}$ oxides has been studied ${ }^{48}$ and was found to be large. We expect that this will also be true for $\mathrm{PuO}_{2}$. However, the covalency is entirely in the closed shells and these shells are spectators for the $\mathrm{M}_{4,5}$ XANES. Hence, we do not consider the $\mathrm{Pu}(6 \mathrm{~d})$ covalency. If we were concerned with the XANES of either the $L_{2,3}$ or $M_{2,3}$ edges, then the $\mathrm{Pu}(6 \mathrm{~d})$ covalency would be critical to the observed splittings as they are for the $\mathrm{U} \mathrm{L}_{3} \mathrm{XAS}$ in $\mathrm{U}$ oxides. ${ }^{10}$

Note that we use four measures of covalent mixing rather than a single measure. The most important consideration is not the precise value of the atomic assignments but rather trends or changes between different orbitals and different compounds. The combination of those four methods is applied here for the first time to evaluate the level of covalency of the An-ligand chemical bond.

Wavefunction Properties. In addition to the character of the orbitals, it is important to understand the character of the $\mathrm{N}$-electron WF for the cluster model of the compound. It is common to describe the $\mathrm{N}$-electron WF by a configuration. Thus, for example, it is tempting to describe the ground state of the $5 \mathrm{f}^{4}$ ground state of $\mathrm{PuO}_{2}$ in terms of a single configuration where all four electrons are in the lowest-energy $\gamma_{8}$ orbital (cf. Figure 1A). Since a $\gamma_{8}$ orbital can only accommodate four electrons, this would be a closed-shell $\gamma_{8}(4)$ configuration with total symmetry $\Gamma_{1}$. With this configuration, $\mathrm{PuO}_{2}$ would not have a permanent magnetic moment. Indeed, $\mathrm{PuO}_{2}$ does not have a permanent moment (cf. ref 53 and references therein), but, as we shall show below, the ground-state WF cannot be represented by the single configuration, but it is a sum of many different configurations. We need to be able to describe, in a compact way, the multiconfiguration WFs for this ground state as well as for the excited states. We know that the WFs will be multiplets with several states having the same energy. However, this is not sufficient to distinguish whether the multiplet is dominated by a single configuration or is a sum of many different configurations where the latter is described as a many-electron effect (cf. ref 36 and references therein).

For the N-electron WFs, it is possible to define orbital occupations, $N(i)$, as a measure of the many-body character of the WFs. The occupation, $N(i)$, is simply the expectation value of the number operator for an orbital in a CI WF. ${ }^{54}$ The $N(i)$ occupation numbers are identical for the WFs of all of the degenerate states in a multiplet. ${ }^{48,54}$ If, for a given multiplet, $N(i)$ is an integer, then WF is described by a single configuration. Significant departures from integral character indicate that different configurations mix to describe the WFs for this multiplet. When we describe the ground- and excited-state WFs for $\mathrm{PuO}_{2}$, we shall use the orbital occupation numbers to characterize the many-electron character of the WFs. This is important since it shows that the $\mathrm{M}_{4,5}$ edge excited states cannot be described as the excitation of an electron from a core orbital into a $5 \mathrm{f}$ orbital. We stress that an important consequence of this multiconfigurational character of the excited states is that the transition intensity is distributed over many different $\mathrm{N}$-electron excited states.

Dipole Intensities. Relative values of the dipole intensities to different final, excited configuration multiplets are obtained from many-electron matrix elements of $\mathbf{r}$ between the WFs for the initial multiplet and the excited multiplet. Sums and averages must be taken over the degenerate WFs of the initial and final multiplets. ${ }^{55}$ The first excited initial multiplet for $\mathrm{Pu}^{4+}$ is at $0.56 \mathrm{eV}$, and for $\mathrm{PuO}_{2}$, it is at $0.12 \mathrm{eV}$, and the Boltzmann occupations of the excited multiplets at room temperature are negligible. The relative intensity to a particular final multiplet, $F$, denoted as $I_{\text {rel }}(F)$ is taken as 


$$
I_{\text {rel }}(F)=\sum_{i, j} \mid<\psi_{i}(\text { initial, } I)|\mathbf{r}| \psi_{j}(\text { final }, F)>\left.\right|^{2}
$$

where the sums over $i$ and $j$ are over the degenerate states of the initial multiplet and the final, excited, multiplet, ${ }^{55}$ and $\Psi_{i}$ and $\Psi_{j}$ are the degenerate CI WFs over individual determinants for specific occupations of the open-shell orbitals; these WFs have been variationally determined.

Since different sets of orbitals are used to describe the $\Psi_{i}$ and $\Psi$ $W F s$, the expression for the matrix elements between individual determinants in the CI expansions of the WFs is more complicated than the case when the two WFs are expanded in the same set of orbitals. $^{56}$ The exact calculation of eq 3 and the neglect of the excitation energy in eq 3 are described in the Supporting Information. Finally, we point out that $I_{\text {rel }}$ in eq 3 is computed for excitations in both $\mathrm{Pu}^{4+}$ and $\mathrm{PuO}_{2}$. The intensities of the theoretical $\mathrm{Pu} \mathrm{M}_{4,5}$ edge XANES spectra were computed with the CLIPS program system. ${ }^{5}$

\section{EXPERIMENTAL SECTION}

The $\mathrm{Pu} \mathrm{M}_{4,5}$ edge HR-XANES experiments were performed at the CAT-ACT beamline at the Karlsruhe research accelerator (KARA), Karlsruhe Institute of Technology (KIT), Karlsruhe Germany. ${ }^{58}$ The incident beam was monochromatized by a $\mathrm{Si}(111)$ double-crystal monochromator (DCM), and focused to a spot size of $500 \mu \mathrm{m} \times 500$ $\mu \mathrm{m}$ onto the sample. The emitted fluorescence was analyzed using a Johan-type X-ray emission spectrometer with Rowland cycle focusing geometry including one spherically bent $\mathrm{Si}(220)$ crystal (SaintGobain Crystals, France) with $1 \mathrm{~m}$ bending radius and a single-diode VITUS silicon drift detector (KETEK, Germany). The X-ray emission spectrometer and the sample were placed inside a glovebox maintaining a constant $\mathrm{He}$ flow so that less than $0.1 \% \mathrm{O}_{2}$ was present in the box during the measurements. This was necessary to minimize intensity loss due to scattering and absorption of the 3-4 $\mathrm{keV}$ X-ray photons. To calibrate the DCM, the energy of the main absorption feature was set to $3970 \mathrm{eV}$ for the $\mathrm{Pu} \mathrm{M}_{4}$ edge and 3775 $\mathrm{eV}$ for $\mathrm{Pu} \mathrm{M} \mathrm{M}_{5}$ edge HR-XANES of $\mathrm{PuO}_{2}$. The spectrometer was focused to the center of mass of the normal emission line measured at $4000 \mathrm{eV}\left(\mathrm{M}_{4}\right)$ or $3800 \mathrm{eV}\left(\mathrm{M}_{5}\right)$ excitation energy and calibrated to $3351 \mathrm{eV} \mathrm{Pu} \mathrm{M}{ }_{\beta}$ or $3534 \mathrm{eV} \mathrm{Pu} \mathrm{M}$. The HR-XANES spectra were collected at incident energies in the range at least of $10 \mathrm{eV}$ before and $30 \mathrm{eV}$ after the edge with a step size of $0.1 \mathrm{eV}$. No beam-induced changes were detectable even after hours of irradiation. The experimental energy resolution was estimated to be $0.7 \mathrm{eV}$. The $\mathrm{PuO}_{2}$ sample was prepared from powdered, bulk ${ }^{239} \mathrm{PuO}_{2}$ and was encapsulated by a $13 \mu \mathrm{m}$ thick Kapton foil (polyimide film $0.013 \mathrm{~mm}$, Advent Research Materials, England) for the first containment and 8 $\mu \mathrm{m}$ Kapton foil to achieve an independent second containment.

\section{RESULTS AND DISCUSSION}

5f based Orbitals for $\mathrm{Pu}^{4+}$ and $\mathrm{PuO}_{2}$ and Their Covalency. For $\mathrm{Pu}^{4+}$, the SO splitting of the DHF orbital energies $(\varepsilon)$ for $\mathrm{M}_{4}\left(3 \mathrm{~d}_{3 / 2}\right)$ and $\mathrm{M}_{5}\left(3 \mathrm{~d}_{5 / 2}\right)$ is $200.0 \mathrm{eV}$, while for the open $5 f$ shell, the SO splitting is $\varepsilon\left(5 f_{7 / 2}\right)-\varepsilon\left(5 f_{5 / 2}\right)=$ $1.0 \mathrm{eV}$; see Figure 1. Clearly, the $3 \mathrm{~d}$ SO splitting is so large that, for the excited-state WFs, there will not be any mixing of configurations with $\mathrm{M}_{4}$ and $\mathrm{M}_{5}$ excitations. However, the $5 \mathrm{f} \mathrm{SO}$ splitting, while it is small, is also comparable to ligand field splittings. For the $\mathrm{O}_{\mathrm{h}}$ geometry of $\mathrm{PuO}_{2},{ }^{31}$ the $\mathrm{Pu} 5 \mathrm{f}$ is ligand field split into $t_{2 w}, t_{1 w}$, and $a_{2 u}$ symmetries (cf. Figure 1A). ${ }^{32}$ From calculations for the embedded $\mathrm{PuO}_{8}$ cluster where only scalar relativistic effects are included, the relative orbital energies with $\Delta \varepsilon\left(\mathrm{t}_{2 \mathrm{u}}\right)=0$ are $\Delta \varepsilon\left(\mathrm{t}_{1 \mathrm{u}}\right)=0.12 \mathrm{eV}$ and $\Delta \varepsilon\left(\mathrm{a}_{2 \mathrm{u}}\right)=$ $0.88 \mathrm{eV}$, where these are spin-free $\Delta \varepsilon$. Since these ligand field splittings and the $1.0 \mathrm{eV}$ SO splittings of the $\varepsilon\left(5 \mathrm{f}_{5 / 2}\right)$ and $\varepsilon\left(5 f_{7 / 2}\right)$ for the isolated $\mathrm{Pu}^{4+}$ cation are comparable, it is necessary to take both types of splittings into account and the orbitals are neither pure SO or pure ligand field split orbitals. ${ }^{30,32}$ For the $\mathrm{O}_{\mathrm{h}}$ double group, the symmetries, with Bethe notation, for an $\mathrm{f}$ electron, including both $\mathrm{SO}$ and ligand field splittings, are $\gamma_{6 w} \gamma_{7 w}$ and $\gamma_{8 \mathrm{u}}$ and there are two sets of $\gamma_{7 \mathrm{u}}$ and $\gamma_{8 \mathrm{u}}$ spin orbitals (cf. Figure 1A). The $\gamma_{6}$ and $\gamma_{7}$ symmetries are twofold degenerate, and the $\gamma_{8}$ symmetry is fourfold degenerate, leading to a total number of 14 possible spinors.

The extent of covalent mixing of the open-shell $\mathrm{Pu} 5 \mathrm{f}$ orbitals with $\mathrm{O} 2 \mathrm{p}$ orbitals to form the ligand field split orbitals of the complex is shown by the properties given in Figure 2

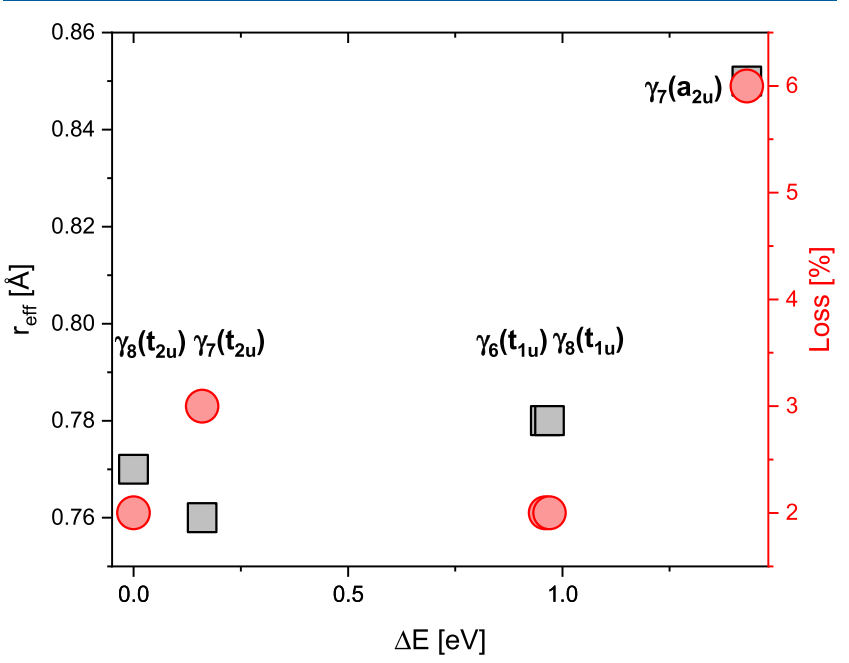

Figure 2. Graphical view of the properties of the $5 f$ orbitals (Table I in the Supporting Information). The energies of the five orbitals $(\Delta \varepsilon)$ are on the abscissa. The size of the orbitals $\left(r_{\text {eff }}\right)$ is shown as gray squares with values on the left $y$ axis, and the loss of $5 f$ character in \% is shown as red circles with values on the right $y$ axis.

and Table I in the Supporting Information. The properties are the relative orbital energies $(\Delta \varepsilon)$, the symmetries of the orbitals, the projections of $\mathrm{Pu} 5 \mathrm{f}$ character, and the sizes of the orbitals as measured by $\mathrm{r}_{\mathrm{eff}}$, where the origin is the Pu nucleus (cf. eq 1). The projections are given as the percent loss of the orbital from the value of 1.00 , which would indicate a pure $\mathrm{Pu}$ orbital. The $\Delta \varepsilon$ values are taken with respect to the lowest orbital energy, which is taken as $\Delta \varepsilon\left(\gamma_{8}\right)=0$ (cf. Figure 1A). The size of the orbital $r_{\text {eff }}$ and the departure of the sum of the $5 f$ projections from 1 (\% loss) are indications of the extent of the covalent mixing of these open-shell orbitals with the ligand $\mathrm{O}(2 \mathrm{p})$ orbitals. An increase in $\mathrm{r}_{\text {eff }}$ over the value for the isolated $\mathrm{Pu}^{4+}$ cation, where $r_{\text {eff }}\left(5 \mathrm{f}_{5 / 2}\right)=0.69 \AA$ and $r_{\text {eff }}\left(5 f_{7 / 2}\right)=$ $0.71 \AA$, indicates a covalent mixing with the ligand orbitals; see eq 1 and related discussion. Larger values of $r_{\text {eff }}$ indicate greater covalency (cf. refs 20,42). The lower-lying four $\mathrm{PuO}_{2}$ orbitals in Figure 2 have similar values of $r_{\text {eff }}$ and \% loss. Their $r_{\text {eff }}$ values are larger than for the isolated cation by $\sim 10 \%$, and they have small losses of $2-3 \%$ from the pure Pu $5 f$ character. This is a direct proof that these orbitals have only minor covalent mixing with the $\mathrm{O}(2 \mathrm{p})$ orbitals.

On the other hand, the highest-lying open-shell orbital with $\Delta \varepsilon=1.43 \mathrm{eV}$ has the largest $\mathrm{r}_{\text {eff }}$ larger than that for the cation by $20 \%$, and it has a loss of pure $5 \mathrm{f}$ character greater than that for the lower-lying orbitals by factors of 2 to 3 . These greater increases indicate that there is more covalent character for this orbital. The fact that we have these three measures, $\Delta \varepsilon$, $\mathrm{r}_{\text {eff }}$ and $\% N_{\mathrm{P}}(5 \mathrm{f})$ loss, which all give a similar analysis of covalent character, is strong evidence for our assignment of the different magnitude of covalent character for the different $5 \mathrm{f}$ orbitals. It 
is important to recognize that the closed-shell orbitals also have covalent character, which, however, is bonding. ${ }^{46}$ Since these orbitals are all doubly occupied, the projected $5 f$ character in the closed shells can be expressed as a number of $5 f\left(5 f_{5 / 2}+5 f_{7 / 2}\right)$ electrons, which is 0.77 . Thus, while any given closed-shell orbital has only very limited $5 f$ character, the sum is modestly large. Since these orbitals are fully occupied in both the ground and excited multiplet WFs, their covalency does not change the relative values of the XANES intensities.

From Figure 2 (Table I in the Supporting Information), the lowest two orbitals, $\gamma_{8}$ and $\gamma_{7}$, are split by $0.16 \mathrm{eV}$. In the limit of large ligand field splitting compared to SO splitting, these orbitals correspond to the $t_{2 u}$ orbital for the spin-free case, and in the limit of large SO splitting, they correspond to the $5 f_{5 / 2}$ orbital of the free cation (cf. Figure 1A). The projections in Table I in the Supporting Information show that these orbitals are indeed dominated by $5 f_{5 / 2}$. The three higher orbitals are dominated by $5 f_{7 / 2}$, but in the limit of large ligand field splitting, the middle $\gamma_{6}$ and $\gamma_{8}$ correspond to the spin-free $t_{1 u}$ and the highest $\gamma_{7}$ orbital corresponds to the most antibonding $\mathrm{a}_{2 \mathrm{u}}$. Since this $\gamma_{7}$ is the most strongly antibonding, it has the largest, 6\%, loss of $5 \mathrm{f}$ character although this is not an especially large loss. This is also the orbital with the largest $r_{\text {eff }}$ $=0.85 \AA$, which is about $10 \%$ larger than the $r_{\text {eff }}$ for the other orbitals. These small increases indicate a small covalent character for the open-shell $\mathrm{PuO}_{2}$ orbitals compared, for example, with the covalent character of the $3 \mathrm{~d}$ orbitals in $\mathrm{Fe}_{2} \mathrm{O}_{3}{ }^{42}$ The covalent character is largest for the highest energy $\gamma_{7}$ orbital. For $U$ oxides, we have shown that the covalent mixing of $\mathrm{U} 5 \mathrm{f}$ and $\mathrm{O}(2 \mathrm{p})$ decreases as the nominal $5 \mathrm{f}$ shell occupation increases. ${ }^{44,48}$ This is consistent with our results for $\mathrm{PuO}_{2}$ which has a large nominal occupation of $45 \mathrm{f}$ electrons. The data in Figure 2 indicate that the $5 \mathrm{f}$ orbitals of $\mathrm{PuO}_{2}$ have only small departures from the character of the $\mathrm{Pu}^{4+}$ orbitals.

The covalent mixing has been viewed as being driven by the overlap of the $5 \mathrm{f}$ orbitals of the $\mathrm{Pu}^{4+}$ cation and the $2 \mathrm{p}$ orbitals of $\mathrm{O}^{2-}$ modified by the energy splitting of these orbitals. ${ }^{50} \mathrm{We}$ have examined the overlaps between the orbitals of $\mathrm{O}(2 \mathrm{p})$, symmetrized to $\mathrm{O}_{8}$, and those of the $\mathrm{Pu}^{4+} 5 f$ orbitals, more precisely the square of the overlaps, $S(i)^{2}$, see eq 2 , to identify the extent that they are consistent with the measures that we have described above. The overlaps are taken for the orbitals without spin-orbit splitting, and the index $i$ is for $t_{1 w}, t_{2 w}$ and $a_{2 u}$ (cf. Figure 1A). For $t_{2 u}$, there are two different $\mathrm{O}_{8}(2 \mathrm{p}$ ) orbitals, and hence two $S^{2}\left(\mathrm{t}_{2 \mathrm{u}}\right)$. The $\mathrm{a}_{2 \mathrm{u}}$ has the largest $S^{2}$ of 0.0085 , the sum of the two $t_{2 u} S^{2}$ is the smallest with 0.0024 , and the $\mathrm{t}_{1 \mathrm{u}} S^{2}$ is intermediate with 0.0038 . This is consistent with the ordering of the spin-free $\mathrm{PuO}_{8}$ orbital energies, but it does not allow us to predict the splitting of the spin-free $\varepsilon$. Thus, we give an estimate only of the orbital overlap driven covalency, which also suggests that $\mathrm{a}_{2 \mathrm{u}}$ has the largest covalent character (cf. Figure 2). Note that we have not included a term for the energy difference of the fragment orbitals since this is not easily available from our $a b$ initio calculations.

The equivalent properties, as those in Figure 2 (Table I in Supporting Information), for the orbitals optimized for the $\mathrm{M}_{4}$ $\rightarrow 5 \mathrm{f}$ and $\mathrm{M}_{5} \rightarrow 5 \mathrm{f}$ excitations are in Figures 3 and 4 (Tables II and III in the Supporting Information), respectively. There are differences from the properties of the ground-state orbitals since the valence open shell has a different occupation and the valence electrons see a different potential because of the presence of a core hole. However, the difference of the

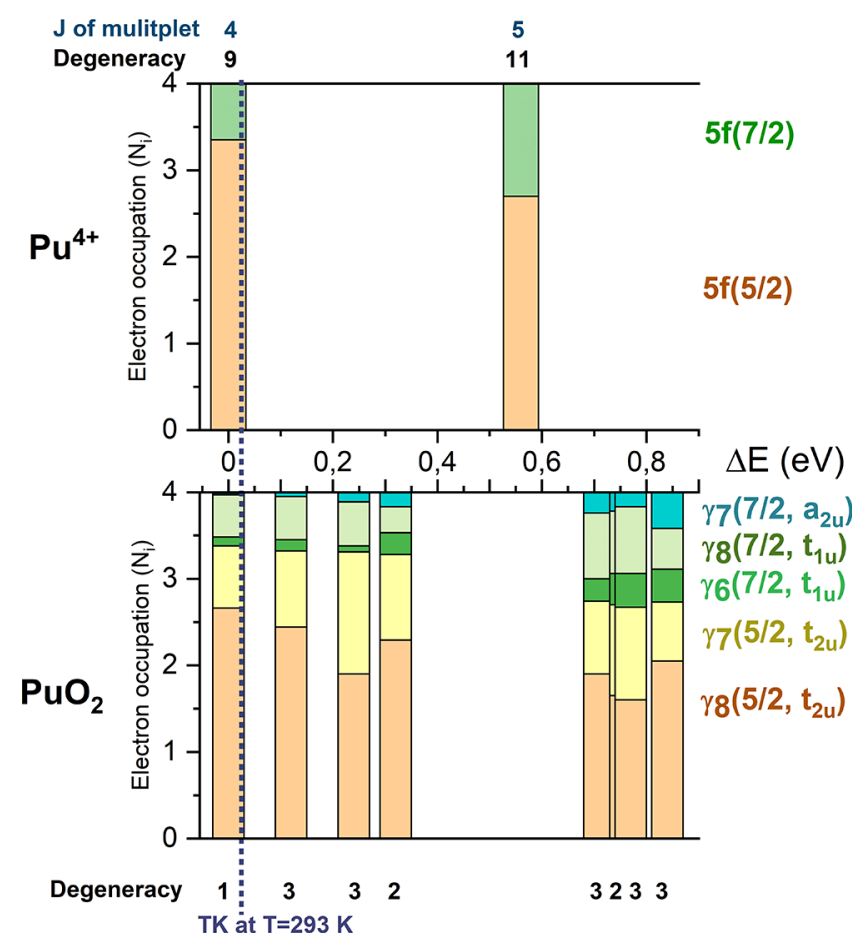

Figure 3. Graphical view of the properties of the lowest multiplets of isolated $\mathrm{Pu}^{4+}$ (top) and of $\mathrm{PuO}_{2}$ (bottom).

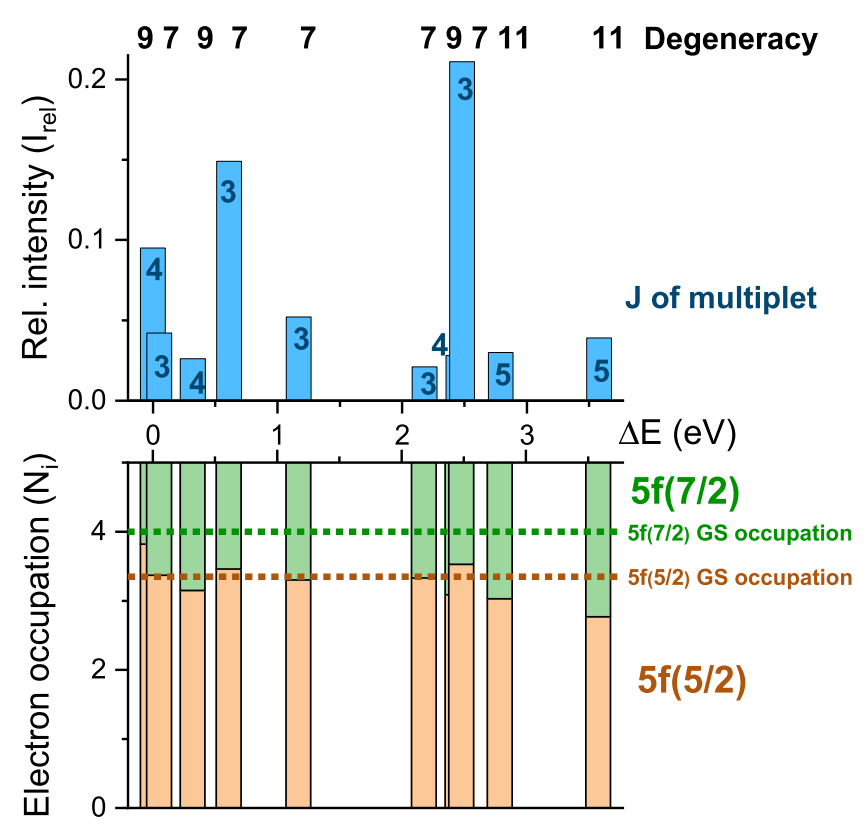

Figure 4. Graphical view of the properties of the 10 multiplets of the $\mathrm{M}_{4} \rightarrow 5 \mathrm{f}$ excited configuration that have the largest XANES intensities $I_{\text {rel }}$. (Top) Intensites of these multiplets together with their $\mathrm{J}$ values; (bottom) contributions of the $5 f_{5 / 2}$ and $5 f_{7 / 2}$ occupations to the total occupation of 5 for the open shell.

properties of the ground-state and excited-state valence openshell orbitals are relatively minor and they have the same general properties. In particular, there is also a very minor covalent mixing for the excited states. This result is different from the conclusion made by Sergentu et al. ${ }^{11}$ for the actinyls $\mathrm{NpO}_{2}{ }^{2+}$ and $\mathrm{PuO}_{2}{ }^{2+}$. These authors found, by natural bond orbital (NBO) analyses, that the mixing of $\mathrm{Pu} / \mathrm{Np}$ and $\mathrm{O} 2 \mathrm{p}$ valence orbitals and the bond order decrease in the excited 
state of the molecule for An $\mathrm{M}_{5}$ absorption edges. As a result, it was concluded that the covalency of the $\mathrm{O}-\mathrm{An}-\mathrm{O}$ axial bond decreases in the excited compared to the ground state of the molecules.

In addition to the similar covalency in the ground-state configuration and the $\mathrm{M}_{4,5}$ configurations, we also find that the highest-energy $\gamma_{7}$ orbital has the largest mixing of $\mathrm{Pu} 5 \mathrm{f}$ with $\mathrm{O}$ $2 \mathrm{p}$ having only 93 or $94 \% \mathrm{Pu}$ character. This suggests that the atomic character of the excitations will dominate the $M_{4,5}$ XANES. It will be shown below that, indeed, the spectra for the isolated atom and for the embedded $\mathrm{PuO}_{8}$ cluster model of $\mathrm{PuO}_{2}$ are very similar. The difference with the results of Sergentu et al. ${ }^{11}$ for plutonyl may arise because of the much shorter $\mathrm{Pu}-\mathrm{O}$ bond distance in plutonyl.

Wavefunctions for the Initial and Excited States. Properties of the lowest $2\left(\mathrm{Pu}^{4+}\right)$ or $8\left(\mathrm{PuO}_{2}\right)$ multiplets are given in Figure 3 (Tables IV and $\mathrm{V}$ in the Supporting Information). These are the degeneracies of the multiplets, the relative energy of the multiplet, $\Delta E$, with $\Delta E=0$ for the lowest energy, and the occupations of the ligand field and spin-orbit split open-shell orbitals (cf. Figures 1 and 2). The nonintegral values of the occupation numbers are compelling evidence that these multiplets cannot be described by a single configuration but are mixtures of several configurations each with different orbital occupations.

The multiplet energies for both $\mathrm{Pu}^{4+}$ and $\mathrm{PuO}_{2}$ are sufficiently high that, at room temperature, only the lowestenergy multiplet has a significant Boltzmann occupation. The first four $\mathrm{PuO}_{2}$ multiplets in Figure 3 (Table $\mathrm{V}$ in the Supporting Information) have values that are close to those for the $J=4$ multiplet of the $\mathrm{Pu}^{4+}$ cation in Figure 3 top (Table IV in the Supporting Information). The higher occupation of the $5 / 2$ compared to $7 / 2$ orbitals is clearly visible. The total number of states associated with these four multiplets is nine, which is the degeneracy of the $\mathrm{Pu}^{4+} J=4$ multiplet. Clearly, these multiplets for $\mathrm{PuO}_{2}$ are ligand field split multiplets of the lowest, $J=4$, multiplet of the $\mathrm{Pu}^{4+}$ cation. The second set of four $\mathrm{PuO}_{2}$ multiplets, which span a $\Delta E$ of $0.7-0.8 \mathrm{eV}$ and are roughly $0.5 \mathrm{eV}$ higher in energy than the first set of four multiplets, also have similar total $5 / 2$ and $7 / 2$ occupations with $\operatorname{Sum}(5 / 2) \approx 2.7$ and $\operatorname{Sum}(7 / 2) \approx 1.3$, respectively, which are very similar to the $5 f_{5 / 2}$ and $5 f_{7 / 2}$ occupations of the excited $\mathrm{Pu}^{4+}$ multiplet (cf. Figure 3 and Supporting Information Tables IV and V). The number of states (11) for this second set are the same as for the first excited, $J=5$, multiplet of the isolated $\mathrm{Pu}^{4+}$ cation. Thus, the second set of $\mathrm{PuO}_{2}$ multiplets are dominantly ligand field split from the $J=5 \mathrm{Pu}^{4+}$ multiplet. The major difference is that the excitation energies, $\Delta E$, for these $\mathrm{PuO}_{2}$ multiplets are $\sim 0.2 \mathrm{eV}$ higher than for the $J=5$ multiplet of $\mathrm{Pu}^{4+}$. These larger values of $\Delta E$ for the excited $\mathrm{PuO}_{2}$ multiplets can be understood from the orbital occupations of these multiplets. The $5 \mathrm{f}$ orbital with the highest $\varepsilon, \mathrm{a}_{2 \mathrm{u}}$ or $\gamma_{7}$ (see Figure 1A) $\Delta \varepsilon \approx 1.5 \mathrm{eV}$ has, as explained in the previous section, the most strong antibonding character of the ligand field split orbitals. The occupation of the orbital, $N\left[\gamma_{7}(7 / 2)\right]$ in Figure 3, is larger for the second group than the first group of states, and this explains the larger excitation energies for $\mathrm{PuO}_{2}$ over $\mathrm{Pu}^{4+}$. Overall, it is possible to view the multiplets of $\mathrm{PuO}_{2}$ as being ligand split multiplets of $\mathrm{Pu}^{4+}$.

Data for the 10 most intense XANES excitations to $M_{4} \rightarrow 5 f$ multiplets for $\mathrm{Pu}^{4+}$ are presented in Figure 4 (and Table VI in Supporting Information). The top histogram gives $I_{\text {rel }}$ and the bottom histogram gives the contributions of the 5 f occupations to the total of five, where different colors, online, are used to distinguish $N\left(5 f_{5 / 2}\right)$ from $N\left(5 f_{7 / 2}\right)$. The common abscissa is the relative energy of the multiplet $(\Delta E)$. On the top histogram, the $J$ values of the multiplets are shown. The contributions of the initial state $5 f$ occupations for the $\Delta E=0$ multiplet in Figure 3 (Table V in the Supporting Information) are shown as dotted lines in the lower histogram. These dotted lines allow us to see how much each of the spin-orbit split $5 \mathrm{f}$ orbitals are occupied by the $\mathrm{M}_{4} \rightarrow$ ff excitation.

It is very clear that the vast majority of the one electron increase in the $5 f$ occupation is into the $5 f_{7 / 2}$ orbital even though the one-electron dipole matrix element between $3 d_{3 / 2}$ and $5 f_{7 / 2}$ is zero. For a few multiplets, the $5 f_{5 / 2}$ occupation is actually smaller than in the initial state multiplet suggesting shake-up-like excitations. For example, for the most intense $\mathrm{M}_{4}$ $\rightarrow 5 \mathrm{f}$ multiplet at $\Delta E=0.61 \mathrm{eV}, N\left(5 \mathrm{f}_{7 / 2}\right)$ increases from the initial state $J=4$ multiplet by 0.9 while the $N\left(5 f_{5 / 2}\right)$ increase is much smaller, only 0.1 . This is compelling visual evidence that one cannot assign the $\mathrm{M}_{4} \rightarrow 5 \mathrm{f}$ excitations as a one-electron process but that one must consider the excitation as a transition between two many-electron multiplets.

The $I_{\text {rel }}$ values are normalized so that the total intensity summed over all multiplets is 1.0; thus, $I_{\text {rel }}$ for a given multiplet is the fraction of the total $\mathrm{M}_{4} \rightarrow 5 \mathrm{f}$ intensity that this multiplet receives. The energy splittings of many of these multiplets are small compared to the lifetime and experimental broadenings, and they will not be resolved in the measurements. This is likely the case for the first four multiplets with $\Delta E$ between 0.0 and $0.6 \mathrm{eV}$, which have a summed intensity of 0.3 and the sixth through ninth multiplets with $2.2 \leq \Delta E \leq 2.8 \mathrm{eV}$, which also have a summed intensity of 0.3 . The total intensity of these 10 multiplets is 0.69 , which means that $31 \%$ of the intensity must arise from multiplets, where each individual has less than $4 \%$ of the total intensity. Although if energy splittings are small, the intensity of several multiplets may combine to give an observable feature. Almost $50 \%$ of the total excitation intensity is distributed largely to the five $J=3$ multiplets in Figure 4 (Table VI in the Supporting Information) with only $20 \%$ going to the other five $J=4$ and 5 multiplets.

The properties of the 10 most intense multiplets for the $M_{5}$ $\rightarrow$ ff excitation for $\mathrm{Pu}^{4+}$ are given in Figure 5 (Table VII in the Supporting Information), which parallels the data in Figure 4 (Table VI in the Supporting Information) for the $\mathrm{M}_{4} \rightarrow 5 \mathrm{f}$ excitations. These intensities have a very different distribution from the $\mathrm{M}_{4} \rightarrow 5 \mathrm{f}$ excitations. The lowest-energy multiplets for this excitation receive much less intensity than for the $M_{4}$ excitation, and the intensities are distributed much more uniformly over a rather small energy range of less than $0.5 \mathrm{eV}$, indicating that they will not be resolved in the measurements. It is not possible to identify states according to the increase of an electron into one of the allowed $5 f_{5 / 2}$ and $5 f_{7 / 2}$ excitations from the ground-state occupations; see $N\left(5 f_{5 / 2}\right)$ and $N\left(5 f_{7 / 2}\right)$ in Figure 5. This is a similar situation as for the $\mathrm{M}_{4}$ excitations where the intensity from the allowed one-electron dipole transition to $5 f_{5 / 2}$ was distributed over many excited-state multiplets mainly with $5 f_{7 / 2}$ character. The total XAS dipole intensity in this group of the 10 most intense multiplets is 59\% of the total intensity, which means that the individual excited multiplets with intensity less than $2.5 \%$ of the total intensity must contribute to the remaining $41 \%$ of the total $M_{5} \rightarrow 5 f$ XAS intensity. Multiplets with $\Delta E<4.16 \mathrm{eV}$ receive $14 \%$, and multiplets with $\Delta E>4.61 \mathrm{eV}$ receive $23 \%$ of this missing $41 \%$ of the XAS intensity; the remainder is distributed to multiplets 


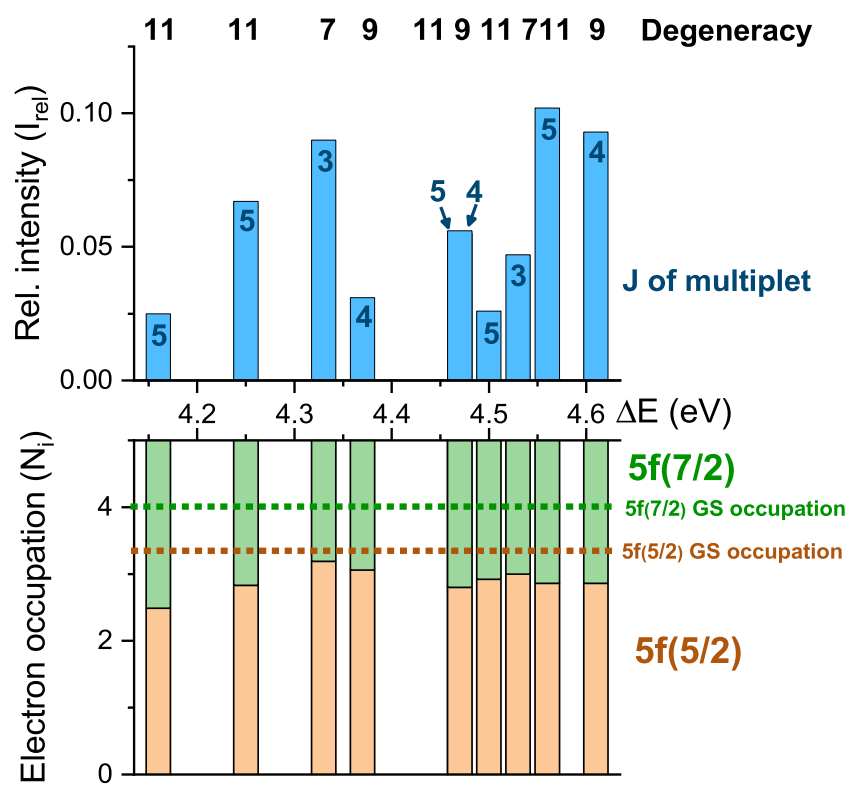

Figure 5. Graphical view of the properties of the 10 multiplets of the $\mathrm{M}_{5} \rightarrow 5 f$ excited configuration that have the largest XANES intensities $I_{\text {rel }}$.

with a low intensity in the energy range of the 10 most intense excited multiplets. As shown in Figure 5 (Table VII in the Supporting Information), the intensity is distributed over $J=3$, 4 , and 5 excited multiplets. Summed over all of the dipoleallowed $\mathrm{M}_{5} \rightarrow$ 5f multiplets, $29.3 \%$ of the XAS intensity goes to $J=3$ multiplets, $35.0 \%$ to $J=4$ multiplets, and $35.7 \%$ to $J=$ 5 multiplets.

For the excitations for $\mathrm{PuO}_{2}$ calculated with the embedded $\mathrm{PuO}_{8}$ cluster, where ligand field splittings are now included, the selection rules are somewhat different and the number of potentially dipole-allowed multiplets increases dramatically. For $\mathrm{PuO}_{2}$, the lowest initial state multiplet is a nondegenerate multiplet with double-group symmetry $\Gamma_{1} \cdot{ }^{30,32}$ Thus, only the excited states with double-group symmetry $\Gamma_{4}$ are dipoleallowed. ${ }^{30}$ For the $\mathrm{M}_{4} \rightarrow$ 5f excited multiplets of $\mathrm{Pu}^{4+}, 90 \%$ of the intensity arises from the 35 multiplets with the largest intensities. However, for the $\mathrm{M}_{4} \rightarrow 5 \mathrm{f}$ excited multiplets of $\mathrm{PuO}_{2}$, it requires 121 of the highest intensity multiplets to obtain $90 \%$ of the total intensity; this is over a factor of 3 larger than for the isolated cation where ligand field splitting is neglected. In other words, including the ligand field splitting dramatically increases the distribution of intensity over a much larger number of excited multiplets. Thus, it is difficult to examine the intensity of individual multiplets for $\mathrm{PuO}_{2}$ as was done for the $\mathrm{M}_{4,5}$, edges of the $\mathrm{Pu}^{4+}$ cation in Figures 4 and 5 (Tables VI and VII in the Supporting Information).

We proceed by explicitly examining how the ligand field splitting in $\mathrm{PuO}_{2}$ changes the distribution of XANES intensity from that for the isolated $\mathrm{Pu}^{4+}$ cation. This is done by plotting the percent fraction of the total intensity for the intensity recovered for all states with relative energy, $\Delta E$, less than $E$ as a function of excitation energy. We denote this intensity as $\operatorname{IR}(E)$, where $\operatorname{IR}(E)=\sum_{\mathrm{i}} \mathrm{I}\left(\Delta E_{i}\right)$, where the sum is over $\mathrm{i}$ for all multiplets with $\Delta E_{i} \leq E$. The $I(\Delta E)$ is the percent of the total XANES intensity for the dipole transition to the excited multiplet with relative energy $\Delta E$. Thus, the value of $\operatorname{IR}(0)$ is the percent intensity for the lowest-energy excited multiplet with $\Delta E=0$. The limiting value of IR for large $E$ is 100 since all excited multiplets are included for large $E$ and the $I_{\text {rel }}$ values are normalized such that the sum of the intensity over all states is 1 . If the distribution of intensity is similar for the isolated $\mathrm{Pu}$ cation and $\mathrm{PuO}_{2}$, then the two curves of $\operatorname{IR}(E)$ will be similar.

The plot in Figure 6A compares IR $(E)$ for the $\mathrm{M}_{4}$ excitation, where the IR for $\mathrm{Pu}^{4+}$ is shown in blue and IR for $\mathrm{PuO}_{2}$ is

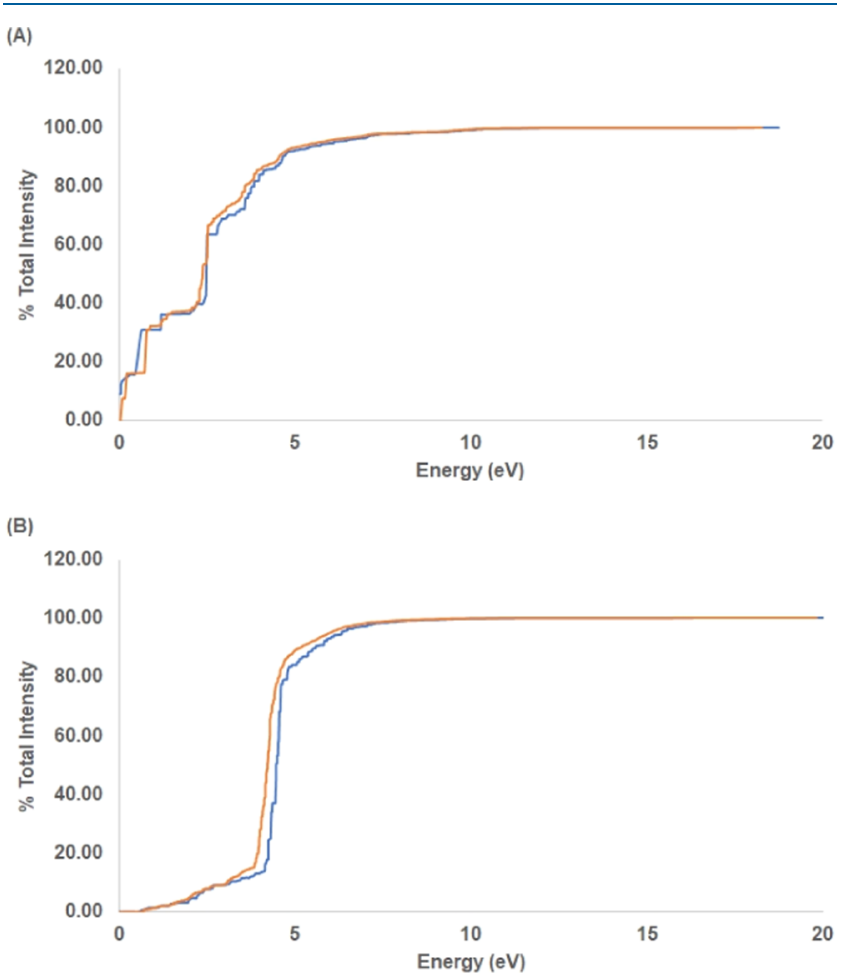

Figure 6. Plots of $\operatorname{IR}(E)$, see eq 1 , obtained for the excitations for $\mathrm{Pu}^{4+}$ (blue curve) and $\mathrm{PuO}_{2}$ (red) curve for $\mathrm{M}_{4} \rightarrow 5$ f excitations (A) and for $\mathrm{M}_{5} \rightarrow 5 \mathrm{f}$ excitations (B).

shown in red. From Figure 6, $\mathrm{Pu}^{4+} \operatorname{IR}(0)=9.5$ as can be seen from the intensity in Figure 4 (see also Table VI in the Supporting Information), while the intensity of the $\mathrm{PuO}_{2}$ $\operatorname{IR}(0)=0$ since the lowest ligand field split $\mathrm{PuO}_{2}$ multiplet does not have $\Gamma_{4}$ symmetry and the first excited multiplet with $\Gamma_{4}$ symmetry is at $\Delta E=0.07 \mathrm{eV}$. However, the two curves are remarkably similar in shape and value; the differences are within the broadening of the measured XAS arising from lifetime and experimental resolution. The equivalent plots of $\operatorname{IR}(E)$ for the $M_{5}$ excitation are in Figure $6 \mathrm{~b}$. Here also, the $\mathrm{Pu}^{4+}$ (blue) and $\mathrm{PuO}_{2}$ (red) $\mathrm{IR}(E)$ are very similar with small energy displacements, usually of $\sim 0.1 \mathrm{eV}$. In other words, the effect of the ligand field splittings on the XAS lead to redistributions of intensity but into multiplets that have small energy separations. The curves in Figure $6 \mathrm{~A}$ for the $\mathrm{M}_{4}$ excitations have a couple of large steps at 0.5 and $2.5 \mathrm{eV}$, which is consistent with the data in Figure 4 (Table VI in the Supporting Information). This would be consistent with a doublet in the XANES that is, indeed, observed; see the following section. On the other hand, the curves in Figure $6 \mathrm{~B}$ for the $M_{5}$ excitations have only a single step at about $4 \mathrm{eV}$.

Comparison of $M_{4,5}$ XANES from Theory and Experiment. The Voigt broadened theory for the $M_{4}$ to $5 f$ and $M_{5}$ to $5 \mathrm{f}$ calculated XANES is compared to the HR-XANES measurements that we have made for these excitations in Figure $7 \mathrm{~A}, \mathrm{~B}$, respectively. The zero of relative excitation 

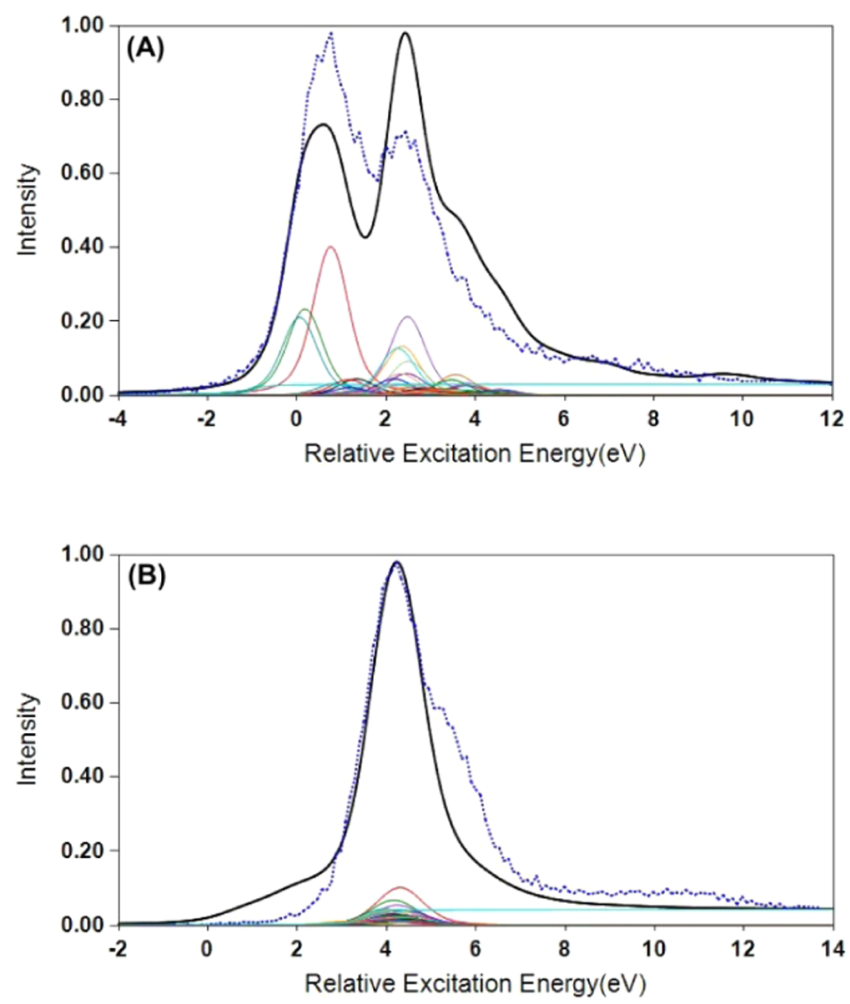

Figure 7. Plots comparing the Voigt broadened theoretical (bold solid curve) and experimental (dotted curve) $\mathrm{Pu} \mathrm{M}_{4}(\mathrm{~A})$ and $\mathrm{M}_{5}$ (B) edge HR-XANES spectra. The most intense individual contributions to the total computed spectrum are also depicted.

energy, $E(\mathrm{rel})=0$, is chosen as the lowest energy excited state. The theoretical excitations are broadened with a Voigt convolution of Lorentzian and Gaussian broadenings ${ }^{59}$ For the Lorentzian lifetime broadening, we have chosen a full width at half-maximum (FWHM) of $0.42 \mathrm{eV}$, which, since we use high-resolution XANES, is much shorter than the $\mathrm{M}_{4}$ or $\mathrm{M}_{5}$ lifetime broadenings of $\mathrm{Pu}$, which are $\approx 4 \mathrm{eV} .^{60}$ The values chosen for the FWHM of the Gaussian broadening to represent experimental resolution are based on matching the leading edge of the rise of the first intense feature from theory and measurement. The choices of the Gaussian FWHM of 0.7 $\mathrm{eV}$ for the $\mathrm{M}_{4}$ edge and $1.0 \mathrm{eV}$ for the $\mathrm{M}_{5}$ edge are discussed below. However, these Gaussian FWHM are similar to the experimental resolution that we estimated in the Experimental Section to be $0.7 \mathrm{eV}$. This close agreement gives us strong confidence in our procedure for broadening our theoretical results. The measured HR-XANES are rigidly shifted so that the measurement and theory are aligned. The intensities of the experiment and theory are scaled so the maximum for both have the value, in arbitrary units, of 0.98 (cf. Figure 7). A continuum step, as an arctan function, ${ }^{61}$ is added to the theory so that the high-energy limit of the theory and the measurement have the same value; the choice of the parameters of the continuum step is empirical. The origin and significance of continuum steps have been reviewed by Stöhr. ${ }^{.1}$ In particular, it argues that it is desirable to account for continuum steps since they can strongly modify the energetic positions and apparent intensities of the near-edge features. ${ }^{61}$ As can be seen from Figure 7, the contribution of the continuum step is small for the $\mathrm{M}_{4,5}$ edges of $\mathrm{PuO}_{2}$. However, we include in the Supporting Information the comparison of theory with experiment where the continuum step is not included but all other broadening parameters are as for the plots of Figure 7. With the exception of the Voigt broadening, the continuum step, and the rigid shift to align theory and experiment, no changes or adjustments of the calculated XAS are made to improve the fit of theory to experiment. Thus, the agreement or disagreement of theory with experiment, as discussed below, provides a proper measure of the completeness of our theory.

There is a reasonable agreement between theory and experiment for the $\mathrm{M}_{4}$ HR-XANES in Figure $7 \mathrm{~A}$, where the theory reproduces the observed doublet although the relative intensities are reversed in the theory. In the low excitation region, $E_{\text {rel }}<-2 \mathrm{eV}$, theory and experiment match rather well, and this is consistent with the choice of Lorentzian broadening to have an FWHM of $0.42 \mathrm{eV}$. The choice of Gaussian broadening with an FWHM of $0.7 \mathrm{eV}$ makes the rise of the theory and experiment for $-1 \leq E_{\text {rel }} \leq 0 \mathrm{eV}$ match very well. The theoretical intensity is somewhat too large compared to experiment in the region around $E_{\text {rel }} \approx 4 \mathrm{eV}$, and this might arise from errors in the multiplet splittings as discussed below. For the $\mathrm{Pu} \mathrm{M}_{5}$ edge HR-XANES, the same Lorentzian, lifetime, broadening of $0.42 \mathrm{eV}$ as for the $\mathrm{M}_{4}$ edge is used and a Gaussian FWHM of $1.0 \mathrm{eV}$ is chosen so that the increase in intensity between $3<E_{\text {rel }}<5 \mathrm{eV}$ for theory and measurement match closely. For this edge, both theory and experiment have a single, modestly broad, peak (cf. Figure 7B). However, the agreement of theory and experiment is not as good as for the $\mathrm{M}_{4}$ edge HR-XANES. The FWHM of the theory is $\sim 1.5 \mathrm{eV}$ compared to the FWHM of the measurement of $\sim 2.2 \mathrm{eV}$. There is also too much intensity for $0<E_{\text {rel }}<3 \mathrm{eV}$ although both theory and experiment have low intensity. Overall, the agreement between theory and measurement is reasonable, but there are significant limitations in the theory and possible origins of these limitations will be identified.

There are several possible limitations in the straightforward theory where only the open-shell angular momentum coupling has been considered (see the Concepts and Methods and Clusters and Wavefunctions sections). A more detailed treatment of many-body effects will give a better description of the multiplet splittings (see, for example, refs 17, 26, 62). For core-level ionized states as observed in XPS, there is a special class of many-body effects that can dramatically change the intensities and energy separations of XPS features. This type of many-body effect has been described as a FAC, or frustrated Auger configuration since a core electron drops to fill the core hole and another electron is promoted to a higher energy or bound level (cf., for example, refs 36, 40 and references therein). Although the FACs have been primarily explored for the XPS of $3 \mathrm{~d}$ transition-metal oxides, ${ }^{63,64}$ such FAC many-body excitations might also be relevant for the $M_{4,5}$ edge XAS of $\mathrm{PuO}_{2}$. For example, there is an atomic effect where an excitation from $6 p$ to $5 f$ occurs in addition to the $3 \mathrm{~d}$ to $5 \mathrm{f}$ excitation. This would be analogous to excitations for the $2 \mathrm{p}$ XPS of transition-metal systems, where, in addition to the metal $2 p$ ionization, there are also excitations of an electron from the $3 \mathrm{~d}$ shell into an $\mathrm{f}$ shell. ${ }^{63,64}$ Inclusion of this type of FAC might lead to further redistribution of intensity and merits investigation.

In addition, there is a possibility of excitation from $M_{4,5}$ to $7 p$ as well as excitations to $5 f$. We have investigated this possibility for the $\mathrm{M}_{5}$ edge of the free $\mathrm{Pu}^{4+}$ cation, and the contribution of the $M_{5}$ to $7 p$ excitations is negligible. This 
follows simply because the $7 \mathrm{p}$ atomic orbital is much more extended than the $5 \mathrm{f}$ orbital. From calculations on the $\mathrm{Pu}^{4+}$ cation, we find that the size, $r_{\text {eff }}$ in eq 1 , for the $7 \mathrm{p}$ orbitals is $\sim 3$ times larger than the $5 \mathrm{f}$ orbitals. This large difference in size means that the magnitude of the dipole transition matrix elements for $3 \mathrm{~d}$ excitation to $7 \mathrm{p},\langle\varphi(3 \mathrm{~d})|\mathbf{r}| \varphi(7 \mathrm{p})\rangle$, will be much smaller than those for $3 \mathrm{~d}$ excitation to the much more compact $5 \mathrm{f},\langle\varphi(3 \mathrm{~d})|\mathbf{r}| \varphi(5 \mathrm{f})\rangle$. Thus, it is unlikely that excitations from $3 \mathrm{~d}$ to $7 \mathrm{p}$ can explain the limitations of the present model.

One last important point is that the HR-XANES spectra are not measured in transmission mode but they are a cross section of a $\mathrm{Pu} 3 \mathrm{~d} 4 \mathrm{f}$ core-to-core resonant inelastic $\mathrm{X}$-ray scattering map ( $3 \mathrm{~d} 4 \mathrm{f}$ CC-RIXS). For a detailed discussion, see ref 12 . In a transmission mode XANES measurement, the core hole in the final state is in the $3 \mathrm{~d}$ level. This is a two-step process including an initial state $\left(3 \mathrm{~d} 105 \mathrm{f}^{4}\right)$ and a final state $\left(3 \mathrm{~d}^{9} 5 \mathrm{f}^{5}\right)$. RIXS is a three-step process; there is an initial $\left(3 \mathrm{~d}^{10} 4 \mathrm{f}^{14} 5 \mathrm{f}^{4}\right)$, intermediate $\left(3 \mathrm{~d}^{9} 4 \mathrm{f}^{14} 5 \mathrm{f}^{5}\right)$ and a final state $\left(3 \mathrm{~d}^{10} 4 \mathrm{f}^{13} 5 \mathrm{f}^{5}\right)$. As a result, the core-hole in the final state is in the $4 \mathrm{f}$ levels of $\mathrm{Pu}$. This adds interactions between the $4 \mathrm{f}$ electrons + core hole with the valence $5 f$ electrons, which are not considered in the calculations. Our result that the $\mathrm{Pu} \mathrm{M}_{4}$ computed spectrum is closer to the experiment compared to the $\mathrm{Pu} \mathrm{M}$ computed spectrum supports this notion. Since the 4f states are split by $13 \mathrm{eV}$ due to spin-orbit coupling, the $4 \mathrm{f}$ electrons + core hole relevant for the $\mathrm{M}_{5}$ edge HR-XANES are much closer to the $5 f$ valence electrons and thus the interactions will be stronger. These effects require further study. Considering that the $\mathrm{a}_{2 \mathrm{u}}$ orbital, which comprises the strongest interaction between metal and ligand orbitals, has the highest energy and $J=7 / 2$ character, excitations for both $M_{4}$ and $M_{5}$ edge to $a_{2 u}$ is expected. It appears that the intensity and energy position of the second peak of the $\mathrm{Pu} \mathrm{M}_{4}$ and the highenergy shoulder in the $\mathrm{Pu} \mathrm{M}_{5}$ edge HR-XANES spectra will be sensitive to variations of the $\mathrm{Pu}$-ligand bonding characteristic.

\section{CONCLUSIONS}

We have presented HR-XANES for the $\mathrm{Pu} \mathrm{M}_{4,5}$ edges and compared these with theoretical predictions for these edges where only excitations from $\mathrm{Pu} 3 \mathrm{~d}$ to $5 \mathrm{f}$ are considered. The theory is based on fully relativistic, four-component, WFs for the ground and $\mathrm{M}_{4,5}$ excited multiplets for an isolated $\mathrm{Pu}^{4+}$ cation and for an embedded cluster model of $\mathrm{PuO}_{2}$. It uses a simple level of the treatment of many-body effects in that only the angular momentum coupling of the core and valence openshell electrons are treated. While additional many-body effects for these multiplets are required to obtain an exact match between theory and experiment, a reasonable agreement between theory and experiment is obtained. This simple level of theory has allowed us to investigate how the XANES intensity for these edges is distributed over the different final multiplets and to understand the proper interpretation of the features of the spectra.

One extremely important conclusion is that one cannot use a one-electron model to interpret the edges. In particular, the WFs for both the initial and final, excited multiplets cannot be represented by a single determinant or by a single open-shell configuration over the spin-orbit and ligand field split orbitals. This is especially true for cases where the initial state is an open-shell system as for the $\mathrm{Pu}(\mathrm{IV})$ systems that we have considered here. However, it is also true that one cannot, in general, use a one-electron model to interpret branching ratios of the core-level XANES of closed-shell systems. ${ }^{65,66}$ Even for excited states of closed-shell systems, one needs to take into account the angular momentum coupling of the electrons in the core shell that has a hole with the excited electron. Only when this coupling is weak, will a one-electron model correctly describe the branching ratios. Thus, for the $\mathrm{O}_{4,5}$ edge of $\mathrm{U}^{+6}$, the one-electron model fails badly, while it is reasonably accurate for the branching ratios of the $\mathrm{N}_{4,5}$ edge; ${ }^{65}$ it is more accurate for the $\mathrm{M}_{4,5}$ edges.

Thus, for open-shell systems, while a one-electron model can indicate that some configurations are dipole-allowed and others are dipole-forbidden, these configurations can mix in the WFs of the multiplets. This mixing is shown through an examination of the occupation numbers of the orbitals, which depart strongly from the integer values that arise for WFs described by a single configuration. The mixing leads to a distribution of the XANES intensity over a large number of states. For this reason, we have had to use a method that includes a full many-electron treatment of the angular momentum coupling. At most, a one-electron model can indicate the shells where excitations will be most intense; thus, it supports the fact that major contributions in the $M_{4,5}$ edges will come from excitations from $3 \mathrm{~d}$ to $5 \mathrm{f}$ and that contributions from $3 \mathrm{~d}$ excitations to the more diffuse $7 \mathrm{p}$, also dipole-allowed, will be less intense.

A second important conclusion is that covalency of the $\mathrm{Pu}-$ $O$ bond does not change between the ground and excited configurations. This allows using the spectra as a tool for detection of the bond covalency for the ground state of the system studied. It is also remarkable that both the $M_{4}$ and $M_{5}$ edge XANES spectra probe mainly $J=5 f(7 / 2)$ states. There is redistribution of electrons, which could be viewed as shake-uplike excitations in the $5 \mathrm{f}$ shell from the lowest orbital energy from $J=5 f(5 / 2)$ into higher orbital energy $J=5 f(7 / 2)$.

A third important conclusion is that the theoretical predictions of the $\mathrm{M}_{4,5}$ edges for the isolated $\mathrm{Pu}$ cation are very similar to those for $\mathrm{PuO}_{2}$. While for the compound the intensity is distributed over a much larger number of excited multiplets than for the isolated cation, the energy splittings and energy shifts of these multiplets for $\mathrm{PuO}_{2}$ compared to those for $\mathrm{Pu}^{4+}$ are small. In particular, they are small compared to the broadenings in the measurements arising from the lifetimes of the excited states and from experimental resolution. In large part, the similarity of the XAS edges for the cation and the compound arises from the relatively small spin-orbit and ligand field splittings of the $5 \mathrm{f}$ shell orbitals compared to the broadening that arises from the angular momentum coupling of the open-shell electrons. The splittings of the $\mathrm{PuO}_{2} 5 \mathrm{f}$ orbitals arising from the combination of ligand field and spinorbit splitting have been examined in terms of the orbital energies, the size of the orbitals, and the projected occupations of atomic orbitals on the $\mathrm{PuO}_{2}$ orbitals. These criteria are consistent with a small covalent mixing of the $\mathrm{Pu}(5 \mathrm{f})$ and $\mathrm{O}(2 \mathrm{p})$ orbitals.

While this mixing is large enough to lead to a nondegenerate $\Gamma_{1}$ symmetry ground-state multiplet for $\mathrm{PuO}_{2},{ }^{53}$ it is not large enough to lead to major changes in the $\mathrm{M}_{4,5}$ edge XANES between $\mathrm{Pu}^{4+}$ and $\mathrm{PuO}_{2}$. However, this is of relevance for $\mathrm{Pu}$ $\mathrm{M}_{4,5}$ edge transmission mode XANES measurements including interactions only between $3 \mathrm{~d}$ and $5 \mathrm{f}$ levels. The HR-XANES comprise in addition $4 \mathrm{f}-5 \mathrm{f}$ interactions, which were not included in the calculations. The $\mathrm{Pu} \mathrm{M}_{4}$ edge HR-XANES are very similar for theory and experiment, suggesting that these 
interactions are less important compared to the $\mathrm{Pu} \mathrm{M}_{5}$ edge HR-XANES case as previously shown. ${ }^{16}$ We see a clear shoulder in the experimental $\mathrm{Pu} \mathrm{M}_{5}$ edge spectra not reproduced by the computations. Since this shoulder very likely contains excitations to the $a_{2 u}$ orbital, which has the highest energy and strongest mixing between $\mathrm{Pu} 5 \mathrm{f}$ and $\mathrm{O} 2 \mathrm{p}$ orbitals, we propose that this energy position and intensity of this shoulder will show sensitivity to changes of chemical bonding of $\mathrm{Pu}$. Similar sensitivity has the second peak in the doublet of the $\mathrm{Pu} \mathrm{M}_{4}$ edge HR-XANES spectrum.

Further investigation to determine the origin of the differences between the predictions of the $M_{4,5}$ edges for the simple many-body model that we have used and the HRXANES measurements is desirable. However, this simple model has provided a firm basis for understanding the main features of these edges. They can be now used for in-depth studies of the role of the An $5 \mathrm{f}$ valence states in chemical bonding. We also demonstrated a set of computational tools for the characterization of bond covalency applicable for any actinide element.

\section{ASSOCIATED CONTENT}

\section{SI Supporting Information}

The Supporting Information is available free of charge at https://pubs.acs.org/doi/10.1021/acs.inorgchem.1c01331.

Specific details of the computation of multiplet WFs (PDF)

\section{AUTHOR INFORMATION}

\section{Corresponding Authors}

Paul S. Bagus - Department of Chemistry, University of North Texas, Denton, Texas 76203-5017, United States; ○ orcid.org/0000-0002-5791-1820; Email: Paul.Bagus@ unt.edu

Tonya Vitova - Karlsruhe Institute of Technology (KIT), Institute for Nuclear Waste Disposal (INE), D-76021 Karlsruhe, Germay; 이이.org/0000-0002-3117-7701; Email: Tonya.Vitova@kit.edu

\section{Author}

Bianca Schacherl - Karlsruhe Institute of Technology (KIT), Institute for Nuclear Waste Disposal (INE), D-76021 Karlsruhe, Germay; (1) orcid.org/0000-0003-4542-0108

Complete contact information is available at: https://pubs.acs.org/10.1021/acs.inorgchem.1c01331

\section{Notes}

The authors declare no competing financial interest.

\section{ACKNOWLEDGMENTS}

P.S.B. gratefully acknowledges support from the U.S. Department of Energy, Office of Science, Office of Basic Energy Sciences, Chemical Sciences, Geosciences, and Biosciences (CSGB) Division through its Geosciences program at Pacific Northwest National Laboratory (PNNL). The authors gratefully acknowledge funding from the European Research Council (ERC) Consolidator Grant 2020 under the European Union's Horizon 2020 research and innovation programme (grant agreement no. 101003292). We thank Steliyana Lehchanska for the design of the graphical art. They thank the Institute for Beam Physics and Technology (IBPT), KIT, for the operation of the storage ring, the Karlsruhe Research Accelerator (KARA).

\section{REFERENCES}

(1) Morgenstern, A.; Apostolidis, C.; Bruchertseifer, F.; Capote, R.; Gouder, T.; Simonelli, F.; Sin, M.; Abbas, K. Cross-sections of the reaction $232 \mathrm{Th}(\mathrm{p}, 3 \mathrm{n}) 230 \mathrm{~Pa}$ for production of $230 \mathrm{U}$ for targeted alpha therapy. Appl. Radiat. Isot. 2008, 66, 1275-1280.

(2) Frantellizzi, V.; Cosma, L.; Brunotti, G.; Pani, A.; Spanu, A.; Nuvoli, S.; De Cristofaro, F.; Civitelli, L.; De Vincentis, G. Targeted Alpha Therapy with Thorium-227. Cancer Biother.Radiopharm. 2020, $35,437-445$.

(3) Morgenstern, A.; Christos, A.; Clemens, K.; Mike, S.; Leszek, K.; Frank, B. An Overview of Targeted Alpha Therapy with 225225Actinium and 213213Bismuth. Curr. Radiopharm. 2018, 11, 200-208.

(4) Vitova, T.; Denecke, M. A.; Göttlicher, J.; Jorissen, K.; Kas, J. J.; Kvashnina, K.; Prüßmann, T.; Rehr, J. J.; Rothe, J. Actinide and lanthanide speciation with high-energy resolution X-ray techniques. $J$. Phys.: Conf. Ser. 2013, 430, No. 012117.

(5) Vitova, T.; Pidchenko, I.; Fellhauer, D.; Pruessmann, T.; Bahl, S.; Dardenne, K.; Yokosawa, T.; Schimmelpfennig, B.; Altmaier, M.; Denecke, M.; Rothe, J.; Geckeis, H. Exploring the electronic structure and speciation of aqueous and colloidal $\mathrm{Pu}$ with high energy resolution XANES and computations. Chem. Commun. 2018, 54, $12824-12827$.

(6) Rothe, J.; Butorin, S.; Dardenne, K.; Denecke, M. A.; Kienzler, B.; Loble, M.; Metz, V.; Seibert, A.; Steppert, M.; Vitova, T.; Walther, C.; Geckeis, H. The INE-Beamline for actinide science at ANKA. Rev. Sci. Instrum. 2012, 83, No. 043105.

(7) Kvashnina, K. O.; Romanchuk, A. Y.; Pidchenko, I.; Amidani, L.; Gerber, E.; Trigub, A.; Rossberg, A.; Weiss, S.; Popa, K.; Walter, O.; Caciuffo, R.; Scheinost, A. C.; Butorin, S. M.; Kalmykov, S. N. A Novel Metastable Pentavalent Plutonium Solid Phase on the Pathway from Aqueous Plutonium(VI) to $\mathrm{PuO} 2$ Nanoparticles. Angew. Chem., Int. Ed. 2019, 58, 17558-17562.

(8) Bahl, S.; Peuge, S.; Pidchenko, I.; Pruessmarm, T.; Rothe, J.; Dardenne, K.; Delrieu, J.; Fellhauer, D.; Jegou, C.; Geckeis, H.; Vitova, T. Pu Coexists in Three Oxidation States in a Borosilicate Glass: Implications for Pu Solubility. Inorg. Chem. 2017, 56, 1398213990.

(9) Bagus, P. S.; Nelin, C. J.; Ilton, E. S. The effect of symmetry on the U L3 NEXAFS of octahedral coordinated uranium(vi). J. Chem. Phys. 2017, 146, No. 114703.

(10) Nelin, C. J.; Bagus, P. S.; Ilton, E. S. Theoretical analysis of the U L3-edge NEXAFS in U oxides. RSC Adv. 2014, 4, 7148-7153.

(11) Sergentu, D.-C.; Duignan, T. J.; Autschbach, J. Ab Initio Study of Covalency in the Ground versus Core-Excited States and X-ray Absorption Spectra of Actinide Complexes. J, Phys. Chem. Lett 2018, 9, 5583-5591.

(12) Vitova, T.; Pidchenko, I.; Fellhauer, D.; Bagus, P. S.; Joly, Y.; Pruessmann, T.; Bahl, S.; Gonzalez-Robles, E.; Rothe, J.; Altmaier, M.; Denecke, M. A.; Geckeis, H. The role of the $5 \mathrm{f}$ valence orbitals of early actinides in chemical bonding. Nat. Commun. 2017, 8, No. 16053.

(13) Thole, B. T.; van der Laan, G. Branching ratio in X-ray absorption spectroscopy. Phys. Rev. B 1988, 38, 3158-3171.

(14) van der Laan, G.; Thole, B. T. X-ray-absorption sum rules in jjcoupled operators and ground-state moments of actinide ions. Phys. Rev. B 1996, 53, 14458-14469.

(15) Moore, K. T.; van der Laan, G. Nature of the $5 f$ states in actinide metals. Rev. Mod. Phys. 2009, 81, 235-298.

(16) Butorin, S. M. 3d-4f Resonant Inelastic X-ray Scattering of Actinide Dioxides: Crystal-Field Multiplet Description. Inorg. Chem. 2020, 59, 16251-16264.

(17) Maganas, D.; Roemelt, M.; Weyhermuller, T.; Blume, R.; Havecker, M.; Knop-Gericke, A.; DeBeer, S.; Schlogl, R.; Neese, F. Ledge $\mathrm{X}$-ray absorption study of mononuclear vanadium complexes and 
spectral predictions using a restricted open shell configuration interaction ansatz. Phys. Chem. Chem. Phys. 2014, 16, 264-276.

(18) Saue, T.; Bast, R.; Gomes, A. S. P.; Jensen, H. J. A.; Visscher, L.; Aucar, I. A.; Remigio, R. D.; Dyall, K. G.; Eliav, E.; Fasshauer, E.; Fleig, T.; Halbert, L.; Hedegård, E. D.; Helmich-Paris, B.; Iliaš, M.; Jacob, C. R.; Knecht, S.; Laerdahl, J. K.; Vidal, M. L.; Nayak, M. K.; Olejniczak, M.; Olsen, J. M. H.; Pernpointner, M.; Senjean, B.; Shee, A.; Sunaga, A.; Stralen, J. N. P. v. The DIRAC code for relativistic molecular calculations. J. Chem. Phys. 2020, 152, No. 204104.

(19) Bagus, P. S.; Nelin, C. J.; Sassi, M. J.; Ilton, E. S.; Rosso, K. M. Analysis of X-Ray Adsorption Edges: L2,3 Edge of FeCl4. J. Chem. Phys. 2017, 147, No. 224306.

(20) Bagus, P. S.; Nelin, C. J.; Sassi, M.; Ilton, E. S.; Rosso, K. M. Consequences of realistic embedding for the L2,3 edge XAS of $\alpha$ Fe2O3. Phys. Chem. Chem. Phys. 2018, 20, 4396.

(21) Cioslowski, J. Density-driven self-consistent-field method: density-constrained correlation energies in the helium series. Phys. Rev. A 1991, 43, 1223-1228.

(22) Valderrama, E.; Ludena, E. V.; Hinze, J. Assessment of dynamical and nondynamical correlation energy components for the beryllium-atom isoelectronic sequence. J. Chem. Phys. 1999, 110, 2343-2353.

(23) Gendron, F.; Autschbach, J. Puzzling Lack of Temperature Dependence of the $\mathrm{PuO} 2$ Magnetic Susceptibility Explained According to $\mathrm{Ab}$ Initio Wave Function Calculations. J, Phys. Chem. Lett 2017, 8, 673-678.

(24) Autschbach, J. Perspective: Relativistic effects. J. Chem. Phys. 2012, 136, No. 150902.

(25) Jung, J.; Islam, M. A.; Pecoraro, V. L.; Mallah, T.; Berthon, C.; Bolvin, H. Derivation of Lanthanide Series Crystal Field Parameters From First Principles. Chem. - Eur. J 2019, 25, 15112-15122.

(26) Hernández, D. P.; Bolvin, H. Magnetic properties of a fourfold degenerate state: $\mathrm{Np} 4+$ ion diluted in $\mathrm{Cs} 2 \mathrm{ZrCl} 6$ crystal. J. Electron Spectrosc. Relat. Phenom. 2014, 194, 74-80.

(27) Slater, J. C. Quantum Theory of Atomic Structure; McGraw-Hill: New York, 1960; Vol. I \& II.

(28) Levine, I. N. Quantum Chemistry; Prentice-Hall: Upper Saddle River, NJ, 2000.

(29) Condon, E. U.; Shortly, G. H. The Theory of Atomic Spectra; Cambridge University Press: Cambridge, 1951.

(30) Altmann, S. L.; Herzig, P. Point-Group Theory Tables; Clarendon Press: Oxford, 1994.

(31) Wyckoff, R. W. G. Crystal Structures; Wiley: New York, 1963.

(32) Boca, R. A Handbook of Magnetochemical Formulae; Elsevier: Amstwerdam, 2012.

(33) Burns, G. Introduction to Group Theory With Applications; Academic Press: New York, 1977.

(34) Bagus, P. S.; Sassi, M. J.; Rosso, K. M. Intermediate Coupling For Core-Level Excited States: Consequences For X-Ray Absorption Spectroscopy. J. Electron Spectrosc. Relat. Phenom. 2015, 200, 174.

(35) Bagus, P. S.; Sassi, M. J.; Rosso, K. M. Cluster embedding of ionic systems: Point charges and extended ions. J. Chem. Phys. 2019, 151, No. 044107.

(36) Bagus, P. S.; Ilton, E. S.; Nelin, C. J. Extracting Chemical Information From XPS Spectra: A Perspective. Catal. Lett. 2018, 148, $1785-1802$.

(37) Visscher, L.; Visser, O.; Aerts, P. J. C.; Merenga, H.; Nieuwpoort, W. C. Relativistic quantum chemistry: The MOLFDIR program package. Comput. Phys. Commun. 1994, 81, 120-144.

(38) Mulliken, R. S. Electronic Population Analysis on LCAO[Single Bond]MO Molecular Wave Functions. I. J. Chem. Phys. 1955, 23, 1833-1840.

(39) Mulliken, R. S. Electronic Population Analysis on LCAO[Single Bond]MO Molecular Wave Functions. II. Overlap Populations, Bond Orders, and Covalent Bond Energies. J. Chem. Phys. 1955, 23, 18411846.

(40) Bagus, P. S.; Ilton, E. S.; Nelin, C. J. The Interpretation of XPS Spectra: Insights Into Materials Properties. Surf. Sci. Rep. 2013, 68, 273.
(41) Dirac, a relativistic ab initio electronic structure program, Release DIRAC14 (2014), written byT., Saue; L., Visscher; H. J., Aa Jensen; R., Bast, with contributions fromV., Bakken; K. G., Dyall; S., Dubillard; U., Ekström; E., Eliav; T., Enevoldsen; E., Faßhauer; T., Fleig; O., Fossgaard; A. S. P., Gomes; T., Helgaker; J., Henriksson; M., Iliaš; Ch. R., Jacob; S., Knecht; S., Komorovský; O., Kullie; C. V., Larsen; J. K., Lærdahl; Y. S., Lee; H. S., Nataraj; P., Norman; G., Olejniczak; J., Olsen; Y. C., Park; J. K., Pedersen; M., Pernpointner; R., di Remigio; K., Ruud; P., Sałek; B., Schimmelpfennig; J., Sikkema; A. J., Thorvaldsen; J., Thyssen; J., van Stralen; S., Villaume; O., Visser; T., Winther; S., Yamamoto (see http://www.diracprogram.org).

(42) Bagus, P. S.; Nelin, C. J.; Brundle, C. R.; Crist, B. V.; Lahiri, N.; Rosso, K. M. Covalency in $\mathrm{Fe} 2 \mathrm{O} 3$ and $\mathrm{FeO}$ : Consequences for XPS Satellite Intensity. J. Chem. Phys. 2020, 153, No. 194702.

(43) Nelin, C. J.; Bagus, P. S.; Philpott, M. R. The nature of the bonding of $\mathrm{CN}$ to metals and organic molecules. J. Chem. Phys. 1987, $87,2170-2176$.

(44) Bagus, P. S.; Nelin, C. J. Covalent Interactions in Oxides. J. Electron Spectrosc. Relat. Phenom. 2014, 194, 37.

(45) Griffith, J. S. The Theory of Transition-Metal Ions; Cambridge Press: Cambridge, 1971.

(46) Ballhausen, C. J. Introduction to Ligand Field Theory; McGrawHill: New York, 1962.

(47) Abragam, A.; Bleaney, B. Electron Paramagnetic Resonance of Transition Ions; Clarendon Press: Oxford, 1970.

(48) Bagus, P. S.; Nelin, C. J.; Hrovat, D. A.; Ilton, E. S. Covalent Bonding In Heavy Metal Oxides. J. Chem. Phys. 2017, 146, No. 134706.

(49) Desclaux, J. P. Relativistic Dirac-Fock expectation values for atoms with $\mathrm{Z}=1$ to $\mathrm{Z}=120$. At. Data Nucl. Data Tables 1973, 12, 311406.

(50) Neidig, M. L.; Clark, D. L.; Martin, R. L. Covalency in felement complexes. Coord. Chem. Rev. 2013, 257, 394-406.

(51) Bagus, P. S.; Nelin, C. J.; Levchenko, S. V.; Zhao, X.; Davis, E. M.; Kuhlenbeck, H.; Freund, H.-J. Surface core level BE shifts for $\mathrm{CaO}(100)$ : insights into physical origins. Phys. Chem. Chem. Phys. 2019, 21, 25431-25438.

(52) Nelin, C. J.; Uhl, F.; Staemmler, V.; Bagus, P. S.; Fujimori, Y.; Sterrer, M.; Kuhlenbeck, H.; Freund, H.-J. Surface core-level binding energy shifts for $\mathrm{MgO}(100)$. Phys. Chem. Chem. Phys. 2014, 16, 21953-21956.

(53) Kern, S.; Robinson, R. A.; Nakotte, H.; Lander, G. H.; Cort, B.; Watson, P.; Vigil, F. A. Crystal-field transition in $\$\{\backslash \operatorname{mathrm}\{\mathrm{PuO}\}\}$

\{2\}\$. Phys. Rev. B 1999, 59, 104-106.

(54) Landau, L. D.; Lifshitz, E. M. Quantum Mechanics; AddisonWesley: Reading, 1958.

(55) Bethe, H. A.; Salpeter, E. W. Quantum Mechanics of One- and Two-Electron Atoms; Academic Press, 1957.

(56) Löwdin, P.-O. Quantum Theory of Many-Particle Systems. I. Physical Interpertations by Means of Density Matrices, Natural SpinOrbitals, and Convergence Problems in the Method of Configurational Interaction. Phys. Rev. 1955, 97, 1474-1489.

(57) CLIPS is a program system to compute ab initio SCF and correlated wavefunctions for polyatomic systems. It has been developed based on the publicly available programs in the ALCHEMY package from the IBM San Jose Research Laboratory by: P. S., Bagus; B., Liu; A. D., McLean; M., Yoshimine.

(58) Zimina, A.; Dardenne, K.; Denecke, M. A.; Doronkin, D. E.; Huttel, E.; Lichtenberg, H.; Mangold, S.; Pruessmann, T.; Rothe, J.; Spangenberg, T.; Steininger, R.; Vitova, T.; Geckeis, H.; Grunwaldt, J.-D. CAT-ACT-A new highly versatile $\mathrm{x}$-ray spectroscopy beamline for catalysis and radionuclide science at the KIT synchrotron light facility ANKA. Rev. Sci. Instrum. 2017, 88, No. 113113.

(59) Gubner, J. A. A new series for approximating Voigt functions. J. Phys. A: Math. Gen. 1994, 27, L745-L749.

(60) Campbell, J. L.; Papp, T. WIDTHS OF THE ATOMIC K - N7 LEVELS. At. Data Nucl. Data Tables 2001, 77, 1-56.

(61) Stöhr, J. NEXAFS Spectroscopy; Springer-Verlag: Berlin, 1992; Vol. 25. 
(62) Gendron, F.; Páez-Hernández, D.; Notter, F.-P.; Pritchard, B.; Bolvin, H.; Autschbach, J. Magnetic Properties and Electronic Structure of Neptunyl(VI) Complexes: Wavefunctions Orbitals, and Crystal-Field Models. Chem. - Eur. J. 2014, 20, 7994-8011.

(63) Bagus, P. S.; Nelin, C. J.; Brundle, C. R.; Crist, B. V.; Lahiri, N.; Rosso, K. M. Combined Multiplet Theory and Experiment for the Fe $2 \mathrm{p}$ and $3 \mathrm{p}$ XPS of $\mathrm{FeO}$ and $\mathrm{Fe}_{2} \mathrm{O}_{3}$. J. Chem. Phys. 2021, 154, No. 094709.

(64) Bagus, P. S.; Broer, R.; Ilton, E. S. Atomic near-degeneracy for photoemission: Generality of $4 \mathrm{f}$ excitations. J. Electron Spectrosc. Relat. Phenom. 2008, 165, 46-49.

(65) Bagus, P. S.; Freund, H.; Kuhlenbeck, H.; Ilton, E. S. A new analysis of X-ray adsorption branching ratios: Use of Russell-Saunders coupling. Chem. Phys. Lett. 2008, 455, 331-334.

(66) van der Laan, G.; Moore, K. T.; Tobin, J. G.; Chung, B. W.; Wall, M. A.; Schwartz, A. J. Applicability of the spin-orbit sum rule for the actinide 5f States. Phys. Rev. Lett. 2004, 93, No. 097401. 